University of South Carolina

Scholar Commons

6-1-2007

\title{
Uniform Estimates for Eulerian-Lagrangian Methods for Singularly Perturbed Time-Dependent Problems
}

\author{
Hong Wang \\ University of South Carolina - Columbia, wang@math.sc.edu \\ Kaixin Wang
}

Follow this and additional works at: https://scholarcommons.sc.edu/math_facpub

Part of the Mathematics Commons

Publication Info

Published in Siam Journal of Numerical Analysis, Volume 45, Issue 3, 2007, pages 1305-1329.

(c) Siam Journal of Numerical Analysis 2007, Society for Industrial and Applied Mathematics Wang, H., \& Wang, K. (2007). Uniform Estimates for Eulerian-Lagrangian Methods for Singularly Perturbed Time-Dependent Problems. SIAM Journal On Numerical Analysis, 45(3), 1305-1329. doi: $10.1137 / 060652816$

This Article is brought to you by the Mathematics, Department of at Scholar Commons. It has been accepted for inclusion in Faculty Publications by an authorized administrator of Scholar Commons. For more information, please contact digres@mailbox.sc.edu. 


\title{
UNIFORM ESTIMATES FOR EULERIAN-LAGRANGIAN METHODS FOR SINGULARLY PERTURBED TIME-DEPENDENT PROBLEMS*
}

\author{
HONG WANG ${ }^{\dagger}$ AND KAIXIN WANG
}

\begin{abstract}
We prove a priori optimal-order error estimates in a weighted energy norm for several Eulerian-Lagrangian methods for singularly perturbed, time-dependent convection-diffusion equations with full regularity. The estimates depend only on certain Sobolev norms of the initial and right-hand side data, but not on $\varepsilon$ or any norm of the true solution, and so hold uniformly with respect to $\varepsilon$. We use the interpolation of spaces and stability estimates to derive an $\varepsilon$-uniform estimate for problems with minimal or intermediate regularity, where the convergence rates are proportional to certain Besov norms of the initial and right-hand side data.
\end{abstract}

Key words. characteristic methods, convergence analysis, error estimates, Eulerian-Lagrangian methods, interpolation of spaces, uniform error estimates

AMS subject classifications. 65M12, 65M25, 65M60, 76M10, 76S05

DOI. $10.1137 / 060652816$

1. Introduction. Time-dependent convection-diffusion equations arise in mathematical models of petroleum reservoir simulation, environmental modeling, and other applications $[10,12]$. These problems admit solutions with moving fronts and complex structures and present serious mathematical and numerical difficulties. Classical finite difference or finite element methods tend to generate numerical solutions with nonphysical oscillations, while upwind methods often produce excessive numerical diffusion that smears out fronts and generates spurious grid orientation effects [10].

Eulerian-Lagrangian methods combine the convection and capacity terms in the governing equations to carry out the temporal discretization in a Lagrangian coordinate and discretize the diffusion term on a fixed mesh $[6,8,16,17,19]$. These methods symmetrize the governing equation and stabilize their numerical approximations. They generate accurate numerical solutions and significantly reduce the numerical diffusion and grid-orientation effect present in upwind methods, even if large time steps and coarse spatial meshes are used. Eulerian-Lagrangian methods were shown to be very competitive in terms of accuracy and efficiency $[6,17,19]$.

Optimal-order error estimates were derived for various Eulerian-Lagrangian methods $[1,7,8,15,18]$. This type of estimates has drawn debates for two potential problems: The general constant may depend inversely on the parameter $\varepsilon$. Further, the smoothness norms of the true solutions on the right side depend inversely on the parameter $\varepsilon$. Consequently, these estimates could blow up as $\varepsilon$ tends to zero.

The goal of the present paper is to derive a priori optimal-order error estimates in an $\varepsilon$-weighted energy norm for Eulerian-Lagrangian methods for singularly perturbed, time-dependent convection-diffusion equations with full regularity. The estimates depend only on certain Sobolev norms of the initial and right-hand side data but not

* Received by the editors February 23, 2006; accepted for publication (in revised form) February 16, 2007; published electronically June 1, 2007.

http://www.siam.org/journals/sinum/45-3/65281.html

${ }^{\dagger}$ School of Mathematics and System Sciences, Shandong University, Jinan, Shandong 250100, China and Department of Mathematics, University of South Carolina, Columbia, SC 29208 (hwang@ math.sc.edu).

$\ddagger$ Corresponding Author. School of Mathematics and System Sciences, Shandong University, Jinan, Shandong 250100, China (kx.wang@mail.sdu.edu.cn). 
on $\varepsilon$ or any norm of the true solution. The general constant in the estimate does not depend on $\varepsilon$ either. Thus, these estimates avoid the problems in the standard estimates. We then use the interpolation of spaces and stability estimates to derive an $\varepsilon$-uniform estimate for problems with minimal or intermediate regularity, where the convergence rates are proportional to certain Besov norms of the initial and right-hand side data.

This paper is organized as follows. In section 2 we recall preliminary results on Sobolev and Besov spaces and interpolation of spaces. In section 3 we revisit the Eulerian-Lagrangian localized adjoint method (ELLAM), the modified method of characteristics (MMOC), and the modified method of characteristics with adjusted advection (MMOCAA). In section 4 we prove an $\varepsilon$-uniform optimal-order error estimate for problems with full regularity. In section 5 we derive $\varepsilon$-uniform error estimates for problems with minimal or intermediate regularity. In section 6 we prove auxiliary lemmas. In section 7 we prove uniform stability of the true solutions in various smoothness norms. Section 8 contains concluding remarks.

2. Model problem and preliminaries. We consider a singularly perturbed, time-dependent convection-diffusion equation in one space dimension

$$
\begin{aligned}
u_{t}+\left(V(x, t) u-\varepsilon D(x, t) u_{x}\right)_{x} & =f(x, t), & (x, t) & \in(a, b) \times(0, T), \\
u(x, 0) & =u_{o}(x), & x & \in[a, b] .
\end{aligned}
$$

Here $V(x, t)$ is a velocity field, $f(x, t)$ accounts for external sources and sinks, $u_{o}(x)$ is prescribed initial data, and $u(x, t)$ is the $\varepsilon$-dependent unknown function. $D(x, t)$ is a diffusion coefficient with

$$
0<D_{\min } \leq D(x, t) \leq D_{\max }<+\infty \quad \forall(x, t) \in[a, b] \times[0, T] .
$$

Here $0<\varepsilon<<1$ is a parameter that scales the diffusion and characterizes the convection dominance of (2.1).

Such Eulerian-Lagrangian methods as the MMOC [8] and the MMOCAA [6, 7] were developed and analyzed for problem (2.1) with periodic boundary conditions. Other methods, such as the ELLAM [3, 18], could handle more general boundary conditions. We analyze these methods in a unified framework and close problem (2.1) with periodic boundary conditions at $x=a$ and $x=b$. This would require that all data functions in the problem are periodic. The assumption of periodicity of the problem may in principle exclude the appearance of boundary layers.

2.1. Sobolev spaces and approximation properties. Let $W_{p}^{k}(a, b)$ consist of functions whose weak derivatives up to order- $k$ are $p$ th Lebesgue integrable in $(a, b)$. Let $H^{k}(a, b):=W_{2}^{k}(a, b)$ and $H_{E}^{1}(a, b)$ be a subspace of $H^{1}(a, b)$ with period $b-a$. We introduce an $\varepsilon$-weighted Sobolev norm for any $v \in H^{k}(a, b)$

$$
\|v\|_{H_{\varepsilon}^{k}(a, b)}:=\left(\|v\|_{H^{k-1}(a, b)}^{2}+\varepsilon\left\|\frac{d^{k} v}{d x^{k}}\right\|_{L^{2}(a, b)}^{2}\right)^{1 / 2} .
$$

For any Banach space $X$, we introduce Sobolev spaces involving time [9]

$$
\begin{aligned}
& W_{p}^{k}\left(t_{1}, t_{2} ; X\right):=\left\{f:\left\|\frac{\partial^{\alpha} f}{\partial t^{\alpha}}(\cdot, t)\right\|_{X} \in L^{p}\left(t_{1}, t_{2}\right), \quad 0 \leq \alpha \leq k, 1 \leq p \leq \infty\right\}, \\
&\|f\|_{W_{p}^{k}\left(t_{1}, t_{2} ; X\right)}:= \begin{cases}\left(\sum_{\alpha=0}^{k} \int_{t_{1}}^{t_{2}}\left\|\frac{\partial^{\alpha} f}{\partial t^{\alpha}}(\cdot, t)\right\|_{X}^{p} d t\right)^{1 / p}, \quad 1 \leq p<\infty, \\
\max _{0 \leq \alpha \leq k} \operatorname{ess} \sup _{\left(t_{1}, t_{2}\right)}\left\|\frac{\partial^{\alpha} f}{\partial t^{\alpha}}(\cdot, t)\right\|_{X}, \quad p=\infty .\end{cases}
\end{aligned}
$$


We define a uniform space-time partition on $[a, b] \times[0, T]: x_{i}:=a+i h$ for $0 \leq i \leq$ $I$, with $h:=(b-a) / I$, and $t_{n}:=n \Delta t$ for $0 \leq n \leq N$, with $\Delta t:=T / N$. If a function $f(x, t)$ is defined only at discrete time steps $t_{n}$, we understand that the function $f$ has been extended by constant to the time interval $\left(t_{n-1}, t_{n}\right]$. Thus, the preceding space-time norm reduces to the following equivalent discrete norm:

$$
\|f\|_{L^{p}(0, T ; X)}:= \begin{cases}\left(\sum_{n=1}^{N}\left\|f\left(\cdot, t_{n}\right)\right\|_{X}^{p} \Delta t\right)^{1 / p}, & 1 \leq p<\infty, \\ \max _{0 \leq n \leq N}\left\|f\left(\cdot, t_{n}\right)\right\|_{X}, & p=\infty .\end{cases}
$$

We also introduce the following $\varepsilon$-weighted energy norms:

$$
\begin{aligned}
\|f\|_{L_{\varepsilon}\left(0, T ; H^{k}(a, b)\right)} & :=\|f\|_{L^{\infty}\left(0, T ; H^{k-1}(a, b)\right)}+\sqrt{\varepsilon}\|f\|_{L^{2}\left(0, T ; H^{k}(a, b)\right)}, \\
\|f\|_{L_{\varepsilon}\left(0, T ; H_{D}^{1}(a, b)\right)} & :=\|f\|_{L^{\infty}\left(0, T ; L^{2}(a, b)\right)}+\sqrt{\varepsilon}\left\|D^{1 / 2} f_{x}\right\|_{L^{2}\left(0, T ; L^{2}(a, b)\right)} .
\end{aligned}
$$

Let $S_{h}(a, b) \subset H_{E}^{1}(a, b)$ be the finite element space that consists of continuous and piecewise-linear functions with respect to the spatial partition in $[a, b]$. We let $\Pi_{h} v \in S_{h}(a, b)$ be the piecewise-linear interpolation of $v$ for any $v \in H_{E}^{1}(a, b)$. The following estimates hold $[4,5]$ :

$$
\begin{array}{rlrl}
\left\|\Pi_{h} v-v\right\|_{H^{k}(a, b)} & \leq C_{1} h^{2-k}\|v\|_{H^{2}(a, b)} & & \forall v \in H^{2}(a, b), k=0,1 \\
\left\|v_{h}\right\|_{H^{1}(a, b)} & \leq C_{2} h^{-1}\left\|v_{h}\right\|_{L^{2}(a, b)} & & \forall v_{h} \in S_{h}(a, b), \\
\left\|v_{h}\right\|_{L^{\infty}(a, b)} \leq C_{2} h^{-1 / 2}\left\|v_{h}\right\|_{L^{2}(a, b)} & & \forall v_{h} \in S_{h}(a, b) .
\end{array}
$$

2.2. Besov spaces and interpolation of operators. The Besov spaces provide a finer scale and characterization of smoothness of functions than the Sobolev spaces do. We cite the results used in this paper and refer readers to $[2,5]$ for details.

For $\alpha>0, k:=\lfloor\alpha\rfloor+1$, and $0<q \leq \infty$, the Besov space $B_{q}^{\alpha}\left(L^{p}(a, b)\right)$ consists of functions $f \in L^{p}(a, b)$ (for $p<\infty$ ) or $f \in C[a, b]$, the space of continuous functions on $[a, b]$, (for $p=\infty)$ such that

$$
\|f\|_{B_{q}^{\alpha}\left(L^{p}(a, b)\right)}:= \begin{cases}\|f\|_{L^{p}(a, b)}+\left[\int_{0}^{\infty}\left[\theta^{-\alpha} \omega_{k}(f, \theta)_{p}\right]^{q} \frac{d \theta}{\theta}\right]^{1 / q}, & 0<q<\infty, \\ \|f\|_{L^{p}(a, b)}+\sup _{\theta>0} \theta^{-\alpha} \omega_{k}(f, \theta)_{p}, & q=\infty,\end{cases}
$$

is finite. Here the $k$ th modulus of smoothness of function $f$ is defined as

$$
\omega_{k}(f, \theta)_{p}:=\sup _{|h| \leq \theta}\left\|\Delta_{h}^{k} f\right\|_{L^{p}(a+k|h|, b-k|h|)} \quad \text { with } \Delta_{h} f(x):=f(x+h)-f(x) .
$$

It is known that $B_{q_{1}}^{\alpha}\left(L^{p}(a, b)\right) \hookrightarrow B_{q_{2}}^{\alpha}\left(L^{p}(a, b)\right)$ for $q_{1}<q_{2}$ and that $B_{2}^{\alpha}\left(L^{2}(a, b)\right)=$ $H^{\alpha}(a, b)$ with equivalent norms.

Let $X_{1} \hookrightarrow X_{0}$ be Banach spaces. We define the $K$-functional for $f \in X_{0}$ by

$$
K(f, s):=K\left(f, s ; X_{0}, X_{1}\right):=\inf _{g \in X_{1}}\left\{\|f-g\|_{X_{0}}+s\|g\|_{X_{1}}\right\}, \quad s \geq 0 .
$$

The interpolation space $\left[X_{0}, X_{1}\right]_{s, q}$ consists of all functions $f \in X_{0}$ such that

$$
\|f\|_{s, q}:= \begin{cases}{\left[\int_{0}^{\infty}\left[\theta^{-s} K(f, \theta)\right]^{q} \frac{d \theta}{\theta}\right]^{1 / q},} & 0<q<\infty, \\ \sup _{\theta>0} \theta^{-s} K(f, \theta), & q=\infty .\end{cases}
$$


It is known that $X_{1} \hookrightarrow\left[X_{0}, X_{1}\right]_{s, q} \hookrightarrow X_{0}$. The following lemmas characterize interpolation spaces.

LEMma 2.1 (interpolation of Sobolev spaces). Let $m$ be a positive integer and $1 \leq p \leq \infty$. For any $0<s<1$ and $0<q \leq \infty$, the following relations hold:

$$
\left[L^{2}(a, b), H^{m}(a, b)\right]_{s, q}=B_{q}^{s m}\left(L^{2}(a, b)\right) .
$$

Lemma 2.2 (interpolation of operators). Let $X_{1} \hookrightarrow X_{0}$ and $Y$ be Banach spaces. If $T$ is a bounded linear operator from $X_{i}$ to $Y$ with norm $M_{i}(i=0,1)$, then $T$ is a bounded linear operator from the interpolation space $\left[X_{0}, X_{1}\right]_{s, q}$ to $Y$ with a norm not exceeding $M_{0}^{1-s} M_{1}^{s}$ for any $0<s<1$ and $0<q \leq \infty$.

LEMMA 2.3 (reiteration theorem). Let $Y_{i}=\left[X_{0}, X_{1}\right]_{s_{i}, q_{i}}(i=0,1)$, with $0<s_{0}<$ $s_{1}<1,0<q_{0}, q_{1} \leq \infty$. For any $0<\beta<1,0<r \leq \infty$, we have

$$
\left[Y_{0}, Y_{1}\right]_{\beta, r}=\left[X_{0}, X_{1}\right]_{\beta^{\prime}, r}, \quad \beta^{\prime}:=(1-\beta) s_{0}+\beta s_{1},
$$

with equivalent norms.

3. Revisit of Eulerian-Lagrangian methods. The ELLAM, MMOC, and MMOCAA schemes use a time-marching approach, so we need only to define these methods at the current time interval $\left[t_{n-1}, t_{n}\right]$.

3.1. The ELLAM. In the ELLAM formulation, the space-time test functions $w(x, t)$ are chosen to be continuous and piecewise smooth and to vanish outside the space-time strip $[a, b] \times\left(t_{n-1}, t_{n}\right]$. In particular, the test functions $w(x, t)$ satisfy $w\left(x, t_{n}\right)=\lim _{t \rightarrow t_{n}-0} w(x, t)$, but $w\left(x, t_{n-1}\right) \neq \lim _{t \rightarrow t_{n-1}+0} w(x, t)$ in general. In this case, we use the notation $w\left(x, t_{n-1}^{+}\right)=\lim _{t \rightarrow t_{n-1}+0} w(x, t)$ to account for the possible discontinuity of $w(x, t)$ in time at time $t_{n-1}$.

We multiply (2.1) by test functions $w$ and integrate the resulting equation on $[a, b] \times\left(t_{n-1}, t_{n}\right]$ to obtain a weak formulation

$$
\begin{array}{rl}
\int_{a}^{b} & u\left(x, t_{n}\right) w\left(x, t_{n}\right) d x+\int_{t_{n-1}}^{t_{n}} \int_{a}^{b} \varepsilon D(x, t) u_{x}(x, t) w_{x}(x, t) d x d t \\
& -\int_{t_{n-1}}^{t_{n}} \int_{a}^{b} u(x, t)\left(w_{t}(x, t)+V(x, t) w_{x}(x, t)\right) d x d t \\
= & \int_{a}^{b} u\left(x, t_{n-1}\right) w\left(x, t_{n-1}^{+}\right) d x+\int_{t_{n-1}}^{t_{n}} \int_{a}^{b} f(x, t) w(x, t) d x d t .
\end{array}
$$

In the ELLAM framework [3] the test functions $w$ are chosen to satisfy the adjoint equation of the hyperbolic part of (2.1) to define the temporal variation of $w$

$$
w_{t}+V w_{x}=0 .
$$

This implies the test functions $w$ to be constant along the characteristic curve $r\left(t ; x, t_{n}\right)$. Here $r(t ; \bar{x}, \bar{t})$ refers to the characteristic curve passing $\bar{x}$ at time $\bar{t}$ defined by

$$
\frac{d r}{d t}=V(r, t),\left.\quad r(t ; \bar{x}, \bar{t})\right|_{t=\bar{t}}=\bar{x} .
$$

Thus, once the test functions $w(x, t)$ are specified in $[a, b]$ at time step $t_{n}$, they are determined completely in the space-time strip $[a, b] \times\left(t_{n-1}, t_{n}\right]$. 
3.1.1. Evaluation of diffusion and source terms. Note that $V(x, t)$ is a periodic function with respect to $x$ of the period $b-a$. Thus, the shifted characteristic curve $r^{S}\left(t ; b, t_{n}\right):=r\left(t ; b, t_{n}\right)-(b-a)$ satisfies the initial-value problem

$$
\begin{array}{ll}
\frac{d r^{S}\left(t ; b, t_{n}\right)}{d t} & =\frac{d r\left(t ; b, t_{n}\right)}{d t}=V\left(r\left(t ; b, t_{n}\right), t\right)=V\left(r^{S}\left(t ; b, t_{n}\right), t\right), \\
\left.r^{S}\left(t ; b, t_{n}\right)\right|_{t=t_{n}} & =b-(b-a)=a .
\end{array}
$$

Therefore, both $r^{S}\left(t ; b, t_{n}\right)$ and $r\left(t ; a, t_{n}\right)$ are the solutions of the same initial-value problem. The uniqueness of such a problem concludes that

$$
r\left(t ; b, t_{n}\right)-r\left(t ; a, t_{n}\right)=b-a \quad \forall t \in\left[t_{n-1}, t_{n}\right] .
$$

For clarity of presentation, in the evaluation of source and diffusion terms we reserve $x$ for points in $[a, b]$ at time $t_{n}$ representing the heads of characteristics. We use the variable $y$ to represent the spatial coordinate of an arbitrary point at time $t \in\left(t_{n-1}, t_{n}\right)$. We use the relation (3.4) and the periodicity of problem (2.1) to evaluate the source term by the Euler quadrature as follows:

$$
\begin{aligned}
& \int_{t_{n-1}}^{t_{n}} \int_{a}^{b} f(y, t) w(y, t) d y d t \\
& \quad=\int_{t_{n-1}}^{t_{n}} \int_{r\left(t ; a, t_{n}\right)}^{r\left(t ; b, t_{n}\right)} f(y, t) w(y, t) d y d t \\
& =\int_{a}^{b} \int_{t_{n-1}}^{t_{n}} f\left(r\left(t ; x, t_{n}\right), t\right) w\left(r\left(t ; x, t_{n}\right), t\right) r_{x}\left(t ; x, t_{n}\right) d t d x \\
& =\int_{a}^{b}\left[\int_{t_{n-1}}^{t_{n}} f\left(r\left(t ; x, t_{n}\right), t\right) r_{x}\left(t ; x, t_{n}\right) d t\right] w\left(x, t_{n}\right) d x \\
& =\Delta t \int_{a}^{b} f\left(x, t_{n}\right) w\left(x, t_{n}\right) d x+E_{1}(w) .
\end{aligned}
$$

Here $E_{1}(w)$ is the local truncation error defined by

$$
E_{1}(w):=\int_{a}^{b} \int_{t_{n-1}}^{t_{n}}\left[f\left(r\left(t ; x, t_{n}\right), t\right) r_{x}\left(t ; x, t_{n}\right)-f\left(x, t_{n}\right)\right] d t w\left(x, t_{n}\right) d x .
$$

We evaluate the diffusion term similarly

$$
\begin{aligned}
& \int_{t_{n-1}}^{t_{n}} \int_{a}^{b} \varepsilon D(y, t) u_{y}(y, t) w_{y}(y, t) d y d t \\
& \quad=\int_{t_{n-1}}^{t_{n}} \int_{r\left(t ; a, t_{n}\right)}^{r\left(t ; b, t_{n}\right)} \varepsilon D(y, t) u_{y}(y, t) w_{y}(y, t) d y d t \\
& =\int_{a}^{b} \int_{t_{n-1}}^{t_{n}} \varepsilon D\left(r\left(t ; x, t_{n}\right), t\right) u_{y}\left(r\left(t ; x, t_{n}\right), t\right) w_{y}\left(x, t_{n}\right) r_{x}\left(t ; x, t_{n}\right) d t d x \\
& =\int_{a}^{b} \int_{t_{n-1}}^{t_{n}} \varepsilon D\left(r\left(t ; x, t_{n}\right), t\right) u_{y}\left(r\left(t ; x, t_{n}\right), t\right) w_{x}\left(x, t_{n}\right) d t d x \\
& =\varepsilon \Delta t \int_{a}^{b} D\left(x, t_{n}\right) u_{x}\left(x, t_{n}\right) w_{x}\left(x, t_{n}\right) d x+\varepsilon E_{2}(u, w) .
\end{aligned}
$$

Here $E_{2}(u, w)$ is the local truncation error defined by

$$
E_{2}(u, w):=\int_{a}^{b} \int_{t_{n-1}}^{t_{n}}\left[\left(D u_{x}\right)\left(r\left(t ; x, t_{n}\right), t\right)-\left(D u_{x}\right)\left(x, t_{n}\right)\right] d t w_{x}\left(x, t_{n}\right) d x .
$$


3.1.2. ELLAM formulation and numerical scheme. We substitute (3.5) and (3.7) into (3.1) to obtain an ELLAM formulation for problem (2.1):

$$
\begin{array}{rl}
\int_{a}^{b} & u\left(x, t_{n}\right) w\left(x, t_{n}\right) d x+\varepsilon \Delta t \int_{a}^{b} D\left(x, t_{n}\right) u_{x}\left(x, t_{n}\right) w_{x}\left(x, t_{n}\right) d x \\
= & \int_{a}^{b} u\left(x^{*}, t_{n-1}\right) w\left(x, t_{n}\right) r_{x}\left(t_{n-1} ; x, t_{n}\right) d x \\
& +\Delta t \int_{a}^{b} f\left(x, t_{n}\right) w\left(x, t_{n}\right) d x+E_{1}(w)-\varepsilon E_{2}(u, w) .
\end{array}
$$

Here $x^{*}$ is the foot of the characteristic curve $r\left(t ; x, t_{n}\right)$ backtracking from $x$ at time $t_{n}$. We also let $\tilde{x}$ be the head of the characteristic curve $r\left(t ; \tilde{x}, t_{n}\right)$ at time $t_{n}$ that backtracks to $x$ at time $t_{n-1}$ :

$$
x^{*}=r\left(t_{n-1} ; x, t_{n}\right), \quad x=r\left(t_{n-1} ; \tilde{x}, t_{n}\right) .
$$

In (3.9) we have used the periodicity of the problem, a relation similar to (3.4), and the fact that $w$ is constant along the characteristics to rewrite the first integral at time $t_{n-1}$ on the right-hand side of (3.1) as an integral at time $t_{n}$ in (3.9):

$$
\begin{aligned}
\int_{a}^{b} u\left(y, t_{n-1}\right) w\left(y, t_{n-1}^{+}\right) d y & =\int_{\tilde{a}}^{\tilde{b}} u\left(x^{*}, t_{n-1}\right) w\left(x, t_{n}\right) r_{x}\left(t_{n-1} ; x, t_{n}\right) d x \\
& =\int_{a}^{b} u\left(x^{*}, t_{n-1}\right) w\left(x, t_{n}\right) r_{x}\left(t_{n-1} ; x, t_{n}\right) d x
\end{aligned}
$$

The ELLAM scheme is derived based on (3.9). Note that the characteristics $r\left(t ; x, t_{n}\right)$ cannot be tracked exactly, in general, so the test functions $w_{h}$ in the ELLAM scheme are defined to be constant along the approximate characteristics $r_{h}\left(t ; x, t_{n}\right)$. Here $r_{h}(t ; \bar{x}, \bar{t})$ is defined by

$$
r_{h}(t ; \bar{x}, \bar{t})=\bar{x}+V(\bar{x}, \bar{t})(t-\bar{t}) .
$$

Consequently, the ELLAM scheme states as follows: Find $u_{h}\left(x, t_{n}\right) \in S_{h}(a, b)$ for $n=1, \ldots, N$ such that for any $w_{h}\left(x, t_{n}\right) \in S_{h}(a, b)$

$$
\begin{aligned}
& \int_{a}^{b} u_{h}\left(x, t_{n}\right) w_{h}\left(x, t_{n}\right) d x+\varepsilon \Delta t \int_{a}^{b} D\left(x, t_{n}\right) u_{h, x}\left(x, t_{n}\right) w_{h, x}\left(x, t_{n}\right) d x \\
& =\int_{a}^{b} u_{h}\left(x_{h}^{*}, t_{n-1}\right) w_{h}\left(x, t_{n}\right) r_{h, x}\left(t_{n-1} ; x, t_{n}\right) d x+\Delta t \int_{a}^{b} f\left(x, t_{n}\right) w_{h}\left(x, t_{n}\right) d x .
\end{aligned}
$$

Here $x_{h}^{*}$ and $\tilde{x}_{h}$ are defined by

$$
x_{h}^{*}=r_{h}\left(t_{n-1} ; x, t_{n}\right), \quad x=r_{h}\left(t_{n-1} ; \tilde{x}_{h}, t_{n}\right) .
$$

The ELLAM, MMOC, MMOCAA, and virtually any other Eulerian-Lagrangian method typically need to impose the following type of constraint on the time step $\Delta t$ :

$$
\|V\|_{L^{\infty}\left(0, T ; W_{\infty}^{1}\right)} \Delta t<1 .
$$

This constraint guarantees that the approximate characteristics defined in (3.12), which are extended from different spatial points, do not intersect with each other during the time period $\left[t_{n-1}, t_{n}\right]$. In other words, the traceback operator defined by the approximate characteristic tracking is a diffeomorphism. This condition will be used several times in the error estimates in the subsequent sections without being explicitly stated. This constraint can be alleviated if a micro time step $\Delta t_{f}$ is used in the characteristic tracking. In this case, the $\Delta t$ in (3.15) will be replaced by $\Delta t_{f}$. 
3.2. The MMOC and MMOCAA. The MMOC and MMOCAA directly apply to a nonconservative analogue of $(2.1)$ :

$$
u_{t}+V(x, t) u_{x}-\left(\varepsilon D(x, t) u_{x}\right)_{x}+V_{x}(x, t) u=f(x, t) .
$$

3.2.1. The MMOC. In the MMOC the capacity and convection terms in (3.16) are combined to form a material derivative at time step $t_{n}$, which is approximated by a backward difference quotient along the approximate characteristic $r_{h}\left(t ; x, t_{n}\right)$ in the time stepping procedure [8]

$$
\begin{aligned}
& u_{t}\left(x, t_{n}\right)+V\left(x, t_{n}\right) u_{x}\left(x, t_{n}\right) \\
&= \sqrt{1+V\left(x, t_{n}\right)^{2}} \frac{d u}{d t}\left(x, t_{n}\right)=\frac{u\left(x, t_{n}\right)-u\left(x^{*}, t_{n-1}\right)}{\Delta t} \\
&+\frac{1}{\Delta t} \int_{t_{n-1}}^{t_{n}} \sqrt{\left(r_{h}\left(t ; x, t_{n}\right)-x_{h}^{*}\right)^{2}+\left(t-t_{n-1}\right)^{2}} \frac{d^{2} u}{d t^{2}}\left(r_{h}\left(t ; x, t_{n}\right), t\right) d t .
\end{aligned}
$$

We incorporate (3.17) into (3.16) and multiply the equation by any test function $w \in H_{E}^{1}(a, b)$. We integrate the resulting equation on the interval $(a, b)$, leading to an MMOC reference equation for problem (3.16): Find $u\left(x, t_{n}\right) \in H_{E}^{1}(a, b)$ for $n=1, \ldots, N$ such that for any $w(x) \in H_{E}^{1}(a, b)$

$$
\begin{aligned}
& \int_{a}^{b} \frac{u\left(x, t_{n}\right)-u\left(x_{h}^{*}, t_{n-1}\right)}{\Delta t} w(x) d x+\int_{a}^{b} \varepsilon D\left(x, t_{n}\right) u_{x}\left(x, t_{n}\right) w_{x}(x) d x \\
& \quad+\int_{a}^{b} V_{x}\left(x, t_{n}\right) u\left(x, t_{n}\right) w(x) d x=\int_{a}^{b} f\left(x, t_{n}\right) w(x) d x-\frac{1}{\Delta t} E_{3}(u, w) .
\end{aligned}
$$

Here $E_{3}(u, w)$ is the local truncation error of the MMOC reference equation

$$
E_{3}(u, w)=\int_{a}^{b} w(x) \int_{t_{n-1}}^{t_{n}} \sqrt{\left(r_{h}\left(t ; x, t_{n}\right)-x_{h}^{*}\right)^{2}+\left(t-t_{n-1}\right)^{2}} \frac{d^{2} u}{d t^{2}}\left(r_{h}\left(t ; x, t_{n}\right), t\right) d t d x .
$$

The MMOC scheme reads: Find $u_{h}\left(x, t_{n}\right) \in S_{h}(a, b)$ for $n=1, \ldots, N$ such that for any $w_{h}(x) \in S_{h}(a, b)$

$$
\begin{aligned}
& \int_{a}^{b} \frac{u_{h}\left(x, t_{n}\right)-u_{h}\left(x_{h}^{*}, t_{n-1}\right)}{\Delta t} w_{h}(x) d x+\int_{a}^{b} \varepsilon D\left(x, t_{n}\right) u_{h, x}\left(x, t_{n}\right) w_{h, x}(x) d x \\
& \quad+\int_{a}^{b} V_{x}\left(x, t_{n}\right) u_{h}\left(x, t_{n}\right) w_{h}(x) d x=\int_{a}^{b} f\left(x, t_{n}\right) w_{h}(x) d x .
\end{aligned}
$$

3.2.2. The MMOCAA. The MMOCAA $[6,7]$ aims at eliminating the mass balance error in the MMOC [8]. Summing the MMOC scheme (3.19) for all of the test functions yields a mass balance satisfied by the MMOC solution $u_{h}\left(x, t_{n}\right)$ :

$$
\int_{a}^{b}\left(1+\Delta t V_{x}\left(x, t_{n}\right)\right) u_{h}\left(x, t_{n}\right) d x=\int_{a}^{b} u_{h}\left(x_{h}^{*}, t_{n-1}\right) d x+\Delta t \int_{a}^{b} f\left(x, t_{n}\right) d x .
$$

If we integrate $(3.16)$ with $u\left(x, t_{n-1}\right)=u_{h}\left(x, t_{n-1}\right)$ on $(a, b) \times\left[t_{n-1}, t_{n}\right]$ and apply Euler quadrature at time $t_{n}$ to the source term, we obtain a mass balance equation satisfied by the exact solution (up to the order of truncation error)

$$
\int_{a}^{b} u\left(x, t_{n}\right) d x=\int_{a}^{b} u_{h}\left(x, t_{n-1}\right) d x+\Delta t \int_{a}^{b} f\left(x, t_{n}\right) d x .
$$


Let $Q_{n-1}=\int_{a}^{b} u_{h}\left(x, t_{n-1}\right) d x$ and $Q_{n-1}^{*}=\int_{a}^{b} u_{h}\left(x_{h}^{*}, t_{n-1}\right) d x-\Delta t \int_{a}^{b} V_{x}\left(x, t_{n}\right)$ $u_{h}\left(x, t_{n}\right) d x$. The MMOC scheme conserves mass if and only if $Q_{n-1}=Q_{n-1}^{*}$. To correct the mass balance error of the MMOC when $Q_{n-1} \neq Q_{n-1}^{*}$, we set for some fixed constant $\kappa>0$

$$
\begin{aligned}
x_{h,+}^{*} & =x_{h}^{*}+\kappa V\left(x, t_{n}\right)(\Delta t)^{2}, \quad x_{h,-}^{*}=x_{h}^{*}-\kappa V\left(x, t_{n}\right)(\Delta t)^{2}, \\
u_{h}^{\#}\left(x_{h}^{*}, t_{n-1}\right) & =\left\{\begin{array}{l}
\max \left\{u_{h}\left(x_{h,+}^{*}, t_{n-1}\right), u_{h}\left(x_{h,-}^{*}, t_{n-1}\right)\right\} \text { if } Q_{n-1}^{*} \leq Q_{n-1}, \\
\min \left\{u_{h}\left(x_{h,+}^{*}, t_{n-1}\right), u_{h}\left(x_{h,-}^{*}, t_{n-1}\right)\right\} \text { if } Q_{n-1}^{*}>Q_{n-1},
\end{array}\right. \\
Q_{n-1}^{\#} & =\int_{a}^{b} u_{h}^{\#}\left(x_{h}^{*}, t_{n-1}\right) d x .
\end{aligned}
$$

If $Q_{n-1}$ can be expressed as a convex combination of $Q_{n-1}^{*}$ and $Q_{n-1}^{\#}$, then $\check{u}_{h}\left(x_{h}^{*}, t_{n-1}\right)$ defined by the same convex combination of $u_{h}\left(x_{h}^{*}, t_{n-1}\right)$ and $u_{h}^{\#}\left(x_{h}^{*}, t_{n-1}\right)$ will have mass $Q_{n-1}$. The $u_{h}\left(x_{h}^{*}, t_{n-1}\right)$ in the MMOC scheme (3.19) is replaced by $\check{u}_{h}\left(x_{h}^{*}, t_{n-1}\right)$ in the MMOCAA scheme with all other terms unchanged.

4. Error estimates for problem (2.1) with full regularity. We prove a priori optimal-order error estimates for the ELLAM, MMOC, and MMOCAA schemes for problem (2.1), which hold uniformly with respect to $\varepsilon$.

4.1. An optimal-order error estimate for the ELLAM scheme. Let the Courant number $\mathrm{Cr}:=\max _{(x, t) \in[0,1] \times[0, T]}|V(x, t)| \Delta t / h$ and $\lambda=1$ if $\mathrm{Cr}<1$ or $=0$ otherwise. The main result is given in the theorem below.

Theorem 4.1. Assume $D, V \in L^{\infty}\left(0, T ; W_{\infty}^{3+\lambda}(a, b)\right), f \in L^{2}\left(0, T ; H^{2+\lambda}(a, b)\right)$, and $u_{o} \in H^{2+\lambda}(a, b)$. Then the following optimal-order error estimate of the ELLAM scheme holds uniformly with respect to $\varepsilon$ :

$$
\begin{aligned}
\left\|u_{h}-u\right\|_{L_{\varepsilon}\left(0, T ; H_{D}^{1}\right)} & \\
\leq & C \Delta t\left(\left\|u_{o}\right\|_{H_{\varepsilon}^{2}}+\left\|\frac{d f}{d t}\right\|_{L^{2}\left(0, T ; L^{2}\right)}+\|f\|_{L^{2}\left(0, T ; H^{1}\right)}\right) \\
& +C\left(\min \{h, \Delta t\}+h^{2}\right)\left\|u_{o}\right\|_{H^{2}}+C \lambda h^{2}\left(\left\|u_{o}\right\|_{H^{3}}+\|f\|_{L^{2}\left(0, T ; H^{3}\right)}\right) .
\end{aligned}
$$

Here the constant $C$ is independent of $u$ and the parameter $\varepsilon$.

Proof. We let $e=u_{h}-u$ and choose the test function $w\left(\cdot, t_{n}\right)$ in $(3.9)$ to be $w_{h}\left(\cdot, t_{n}\right) \in S_{h}(a, b)$. We then subtract (3.13) from the ELLAM reference equation (3.9) to obtain an ELLAM error equation for any $w_{h}\left(x, t_{n}\right) \in S_{h}(a, b)$ :

$$
\begin{aligned}
& \int_{a}^{b} e\left(x, t_{n}\right) w_{h}\left(x, t_{n}\right) d x+\varepsilon \Delta t \int_{a}^{b} D\left(x, t_{n}\right) e_{x}\left(x, t_{n}\right) w_{h, x}\left(x, t_{n}\right) d x \\
& =\int_{a}^{b}\left(u\left(x_{h}^{*}, t_{n-1}\right) r_{h, x}\left(t_{n-1} ; x, t_{n}\right)-u\left(x^{*}, t_{n-1}\right) r_{x}\left(t_{n-1} ; x, t_{n}\right)\right) w_{h}\left(x, t_{n}\right) d x \\
& \quad+\int_{a}^{b} e\left(x_{h}^{*}, t_{n-1}\right) w_{h}\left(x, t_{n}\right) r_{h, x}\left(t_{n-1} ; x, t_{n}\right) d x-E_{1}\left(w_{h}\right)+\varepsilon E_{2}\left(u, w_{h}\right) .
\end{aligned}
$$

Let $\Pi_{h} u \in S_{h}(a, b)$ be the interpolation of the true solution $u, \xi_{h}=u_{h}-\Pi_{h} u \in$ $S_{h}(a, b)$, and $\eta=\Pi_{h} u-u$. The error estimates for $\eta$ are given in (2.3), so we need 
only to estimate $\xi_{h}$. We choose $w_{h}\left(x, t_{n}\right)=\xi_{h}\left(x, t_{n}\right)$ in $(4.2)$ and rewrite the error equation in terms of $\xi_{h}$ and $\eta$ as follows:

$$
\begin{aligned}
& \int_{a}^{b} \xi_{h}^{2}\left(x, t_{n}\right) d x+\varepsilon \Delta t \int_{a}^{b} D\left(x, t_{n}\right) \xi_{h, x}^{2}\left(x, t_{n}\right) d x \\
& =\int_{a}^{b} \xi_{h}\left(x_{h}^{*}, t_{n-1}\right) \xi_{h}\left(x, t_{n}\right) r_{h, x}\left(t_{n-1} ; x, t_{n}\right) d x \\
& \quad+\int_{a}^{b} \eta\left(x_{h}^{*}, t_{n-1}\right) \xi_{h}\left(x, t_{n}\right) r_{h, x}\left(t_{n-1} ; x, t_{n}\right) d x-\int_{a}^{b} \eta\left(x, t_{n}\right) \xi_{h}\left(x, t_{n}\right) d x \\
& \quad-\varepsilon \Delta t \int_{a}^{b} D\left(x, t_{n}\right) \eta_{x}\left(x, t_{n}\right) \xi_{h, x}\left(x, t_{n}\right) d x-E_{1}\left(\xi_{h}\right)+\varepsilon E_{2}\left(u, \xi_{h}\right) \\
& \quad+\int_{a}^{b} u\left(x^{*}, t_{n-1}\right)\left(r_{h, x}\left(t_{n-1} ; x, t_{n}\right)-r_{x}\left(t_{n-1} ; x, t_{n}\right)\right) \xi_{h}\left(x, t_{n}\right) d x \\
& \quad+\int_{a}^{b}\left(u\left(x_{h}^{*}, t_{n-1}\right)-u\left(x^{*}, t_{n-1}\right)\right) r_{h, x}\left(t_{n-1} ; x, t_{n}\right) \xi_{h}\left(x, t_{n}\right) d x .
\end{aligned}
$$

We bound the first term on the right-hand side of (4.3) by

$$
\begin{aligned}
& \left|\int_{a}^{b} \xi_{h}\left(x_{h}^{*}, t_{n-1}\right) \xi_{h}\left(x, t_{n}\right) r_{h, x}\left(t_{n-1} ; x, t_{n}\right) d x\right| \\
& \quad \leq \frac{1+C \Delta t}{2} \int_{a}^{b} \xi^{2}\left(x, t_{n}\right) d x+\frac{1+C \Delta t}{2} \int_{a}^{b} \xi^{2}\left(x_{h}^{*}, t_{n-1}\right) d x \\
& \quad \leq \frac{1+C \Delta t}{2} \int_{a}^{b} \xi^{2}\left(x, t_{n}\right) d x+\frac{1+C \Delta t}{2} \int_{a_{h}^{*}}^{b_{h}^{*}} \xi^{2}\left(x_{h}^{*}, t_{n-1}\right)\left|\frac{d x_{h}^{*}}{d x}\right|^{-1} d x_{h}^{*} \\
& \quad \leq \frac{1+C \Delta t}{2}\left\|\xi_{h}\left(\cdot, t_{n}\right)\right\|_{L^{2}}^{2}+\frac{1+C \Delta t}{2}\left\|\xi_{h}\left(\cdot, t_{n-1}\right)\right\|_{L^{2}}^{2} .
\end{aligned}
$$

Here the constant $C$ depends on $\|V\|_{L^{\infty}\left(0, T ; W_{\infty}^{1}\right)}$. In the second term after the second inequality, we used the substitution of variables from $x$ to $x_{h}^{*}$ given by the first equation in (3.14) and changed the limits $a$ and $b$ of the integral to $a_{h}^{*}$ and $b_{h}^{*}$, respectively. We also utilized (3.12) and the periodicity of $V$ to conclude that

$$
\begin{aligned}
r_{h, x}\left(t_{n-1} ; x, t_{n}\right) & =1-V_{x}\left(x, t_{n}\right) \Delta t, \\
r_{h, x}^{-1}\left(t_{n-1} ; x, t_{n}\right) & =\left(1-V_{x}\left(x, t_{n}\right) \Delta t\right)^{-1}=1+O(\Delta t), \\
b_{h}^{*}-a_{h}^{*} & =(b-a)-\left(V\left(b, t_{n}\right)-V\left(a, t_{n}\right)\right) \Delta t=b-a .
\end{aligned}
$$

The estimate of the second and third terms on the right-hand side of (4.3) presents one of the major difficulties. Standard techniques yield

$$
\begin{aligned}
& \left|\int_{a}^{b}\left(\eta\left(x, t_{n-1}\right)-\eta\left(x_{h}^{*}, t_{n-1}\right)\right) \xi_{h}\left(x, t_{n}\right) d x\right| \\
& \quad=\left|\int_{a}^{b} \int_{x_{h}^{*}}^{x} \eta_{y}\left(y, t_{n-1}\right) d y \xi_{h}\left(x, t_{n}\right) d x\right| \\
& \quad \leq C \Delta t\left\|\xi_{h}\left(\cdot, t_{n}\right)\right\|_{L^{2}}^{2}+C \Delta t h^{2}\|u\|_{L^{\infty}\left(0, T ; W_{\infty}^{2}\right)}^{2}
\end{aligned}
$$

leading to a suboptimal-order error estimate of order $O(h+\Delta t)$ for the ELLAM scheme. This does not coincide with the optimal-order convergence rates observed numerically. A delicate analysis shows an optimal-order error estimate of the second and third terms on the right-hand side of (4.3). For clarity of exposition, the proof is 
presented in Lemma 6.2 ; there we obtain

$$
\begin{aligned}
& \left|\int_{a}^{b} \eta\left(x_{h}^{*}, t_{n-1}\right) \xi_{h}\left(x, t_{n}\right) r_{h, x}\left(t_{n-1} ; x, t_{n}\right) d x-\int_{a}^{b} \eta\left(x, t_{n}\right) \xi_{h}\left(x, t_{n}\right) d x\right| \\
& \quad \leq C \Delta t\left\|\xi_{h}\left(\cdot, t_{n}\right)\right\|_{L^{2}}^{2}+C \Delta t\left(\min \left\{h^{2},(\Delta t)^{2}\right\}+h^{4}\right)\|u\|_{L^{\infty}\left(0, T ; H^{2}\right)}^{2} \\
& \quad+C(\Delta t)^{3}\|u\|_{L^{\infty}\left(0, T ; H^{1}\right)}^{2}+C \lambda h^{4}\left(\|u\|_{H^{1}\left(t_{n-1}, t_{n} ; H^{2}\right)}^{2}+\Delta t\|u\|_{L^{\infty}\left(0, T ; H^{3}\right)}^{2}\right) .
\end{aligned}
$$

Let $x_{i-1 / 2}$ be the middle point of the interval $\left[x_{i-1}, x_{i}\right]$. Note that $\xi_{h, x}\left(x, t_{n}\right)$ is constant on each interval $\left[x_{i-1}, x_{i}\right]$ and that $\eta$ satisfies $\eta\left(x_{i-1}, t_{n}\right)=\eta\left(x_{i}, t_{n}\right)=0$ for $i=1, \ldots, I$. We bound the fourth term on the right-hand side of (4.3):

$$
\begin{aligned}
& \left|\varepsilon \Delta t \int_{a}^{b} D\left(x, t_{n}\right) \eta_{x}\left(x, t_{n}\right) \xi_{h, x}\left(x, t_{n}\right) d x\right| \\
& \quad=\left|\varepsilon \Delta t \sum_{i=1}^{I} \xi_{h, x}\left(x_{i-1 / 2}, t_{n}\right) \int_{x_{i-1}}^{x_{i}}\left(D\left(x, t_{n}\right)-D\left(x_{i-1 / 2}, t_{n}\right)\right) \eta_{x}\left(x, t_{n}\right) d x\right| \\
& \quad \leq \varepsilon \Delta t h\|D\|_{L^{\infty}\left(0, T ; W_{\infty}^{1}\right)}\left\|\xi_{h, x}\left(\cdot, t_{n}\right)\right\|_{L^{2}}\left\|\eta_{x}\left(\cdot, t_{n}\right)\right\|_{L^{2}} \\
& \quad \leq \frac{1}{4} \varepsilon \Delta t\left\|\xi_{h, x}\left(\cdot, t_{n}\right)\right\|_{L_{D}^{2}}^{2}+C \varepsilon \Delta t h^{4}\|u\|_{L^{\infty}\left(0, T ; H^{2}\right)}^{2} .
\end{aligned}
$$

Here $\|\cdot\|_{L_{D}^{2}}=\left\|D^{1 / 2} \cdot\right\|_{L^{2}}$.

We use the estimate (6.1) to bound the fifth term on the right side of (4.3):

$$
\begin{aligned}
& \left|\int_{a}^{b} \int_{t_{n-1}}^{t_{n}}\left[f\left(r\left(t ; x, t_{n}\right), t\right) r_{x}\left(t ; x, t_{n}\right)-f\left(x, t_{n}\right)\right] d t \xi_{h}\left(x, t_{n}\right) d x\right| \\
& \quad \leq \int_{a}^{b} \int_{t_{n-1}}^{t_{n}}\left|f\left(x, t_{n}\right)-f\left(r\left(t ; x, t_{n}\right), t\right)\right| d t\left|\xi_{h}\left(x, t_{n}\right)\right| d x \\
& \quad+\int_{a}^{b} \int_{t_{n-1}}^{t_{n}}\left|f\left(r\left(t ; x, t_{n}\right), t\right)\right|\left|1-r_{x}\left(t ; x, t_{n}\right)\right| d t\left|\xi_{h}\left(x, t_{n}\right)\right| d x \\
& \quad \leq C \Delta t\left\|\xi_{h}\left(\cdot, t_{n}\right)\right\|_{L^{2}}^{2}+C(\Delta t)^{2}\left(\left\|\frac{d f}{d t}\right\|_{L^{2}\left(t_{n-1}, t_{n} ; L^{2}\right)}^{2}+\|f\|_{L^{2}\left(t_{n-1}, t_{n} ; L^{2}\right)}^{2}\right) .
\end{aligned}
$$

We similarly bound the sixth term on the right-hand side of (4.3) by

$$
\begin{aligned}
& \left|\varepsilon \int_{a}^{b} \int_{t_{n-1}}^{t_{n}}\left[\left(D u_{x}\right)\left(x, t_{n}\right)-\left(D u_{x}\right)\left(r\left(t ; x, t_{n}\right), t\right)\right] d t \xi_{h, x}\left(x, t_{n}\right) d x\right| \\
& \quad=\left|\varepsilon \int_{a}^{b} \xi_{h, x}\left(x, t_{n}\right)\left[\int_{t_{n-1}}^{t_{n}} \int_{t}^{t_{n}} \frac{d}{d \theta}\left(D u_{x}\right)\left(r\left(\theta ; x, t_{n}\right), \theta\right) d \theta d t\right] d x\right| \\
& \quad \leq \frac{1}{4} \varepsilon \Delta t\left\|\xi_{h, x}\left(\cdot, t_{n}\right)\right\|_{L_{D}^{2}}^{2}+C \varepsilon(\Delta t)^{2}\left(\left\|\frac{d u}{d t}\right\|_{L^{2}\left(t_{n-1}, t_{n} ; H^{1}\right)}^{2}+\|u\|_{L^{2}\left(t_{n-1}, t_{n} ; H^{1}\right)}^{2}\right) .
\end{aligned}
$$

We use the estimate (6.2) to bound the seventh term on the right side of (4.3) in a similar way to the estimate (4.4) to get

$$
\begin{aligned}
& \left|\int_{a}^{b} u\left(x^{*}, t_{n-1}\right)\left(r_{h, x}\left(t_{n-1} ; x, t_{n}\right)-r_{x}\left(t_{n-1} ; x, t_{n}\right)\right) \xi_{h}\left(x, t_{n}\right) d x\right| \\
& \quad \leq C(\Delta t)^{2}\left\|\xi_{h}\left(\cdot, t_{n}\right)\right\|_{L^{2}}\left(\int_{a}^{b} u^{2}\left(x^{*}, t_{n-1}\right) d x\right)^{1 / 2} \\
& \quad \leq C \Delta t\left\|\xi_{h}\left(\cdot, t_{n}\right)\right\|_{L^{2}}^{2}+C(\Delta t)^{3}\|u\|_{L^{\infty}\left(0, T ; L^{2}\right)}^{2} .
\end{aligned}
$$

Let $\chi_{(\alpha, \beta)}$ be the indicator function of the interval $(\alpha, \beta)$, which is 1 on $(\alpha, \beta)$ or 0 elsewhere. We use the estimate (6.1) to bound the last term on the right-hand side 
of (4.3):

$$
\begin{aligned}
& \left|\int_{a}^{b}\left(u\left(x_{h}^{*}, t_{n-1}\right)-u\left(x^{*}, t_{n-1}\right)\right) r_{h, x}\left(t_{n-1} ; x, t_{n}\right) \xi_{h}\left(x, t_{n}\right) d x\right| \\
& \quad \leq C \int_{a}^{b}\left|\int_{x^{*}}^{x_{h}^{*}}\right| u_{y}\left(y, t_{n-1}\right)|d y|\left|\xi_{h}\left(x, t_{n}\right)\right| d x \\
& \leq C \Delta t\left\|\xi_{h}\left(\cdot, t_{n}\right)\right\|_{L^{2}}\left(\int_{a}^{b} \int_{a}^{b} \chi(y)_{\left(x^{*}-C(\Delta t)^{2}, x^{*}+C(\Delta t)^{2}\right)} u_{y}^{2}\left(y, t_{n-1}\right) d y d x\right)^{1 / 2} \\
& \quad=C \Delta t\left\|\xi_{h}\left(\cdot, t_{n}\right)\right\|_{L^{2}}\left(\int_{a}^{b} \int_{a}^{b} \chi(x)_{\left(y-C(\Delta t)^{2}, y+C(\Delta t)^{2}\right)} d x u_{y}^{2}\left(y, t_{n-1}\right) d y\right)^{1 / 2} \\
& \leq C \Delta t\left\|\xi_{h}\left(\cdot, t_{n}\right)\right\|_{L^{2}}^{2}+C(\Delta t)^{3}\|u\|_{L^{\infty}\left(0, T ; H^{1}\right)}^{2} .
\end{aligned}
$$

We substitute estimates (4.4)-(4.11) for the corresponding terms in (4.3) to obtain the following estimate:

$$
\begin{aligned}
\left\|\xi_{h}\left(\cdot, t_{n}\right)\right\|_{L^{2}}^{2}+\varepsilon \Delta t\left\|\xi_{h, x}\left(\cdot, t_{n}\right)\right\|_{L_{D}^{2}}^{2} & \\
\leq & \frac{1+C \Delta t}{2}\left(\left\|\xi_{h}\left(\cdot, t_{n}\right)\right\|_{L^{2}}^{2}+\left\|\xi_{h}\left(\cdot, t_{n-1}\right)\right\|_{L^{2}}^{2}\right)+\frac{1}{2} \varepsilon \Delta t\left\|\xi_{h, x}\left(\cdot, t_{n}\right)\right\|_{L_{D}^{2}}^{2} \\
& +C(\Delta t)^{2}\left(\varepsilon\left\|\frac{d u}{d t}\right\|_{L^{2}\left(t_{n-1}, t_{n} ; H^{1}\right)}^{2}+\Delta t\|u\|_{L^{\infty}\left(0, T ; H^{1}\right)}^{2}+\left\|\frac{d f}{d t}\right\|_{L^{2}\left(t_{n-1}, t_{n} ; L^{2}\right)}^{2}\right. \\
& \left.\quad+\|f\|_{L^{2}\left(t_{n-1}, t_{n} ; L^{2}\right)}^{2}\right)+C \Delta t \min \left\{h^{2},(\Delta t)^{2}\right\}\|u\|_{L^{\infty}\left(0, T ; H^{2}\right)}^{2} \\
& +C h^{4}\left(\Delta t\|u\|_{L^{\infty}\left(0, T ; H^{2}\right)}^{2}+\lambda\left(\|u\|_{H^{1}\left(t_{n-1}, t_{n} ; H^{2}\right)}^{2}+\Delta t\|u\|_{L^{\infty}\left(0, T ; H^{3}\right)}^{2}\right)\right) .
\end{aligned}
$$

We sum the estimate for $n=1, \ldots, N_{1}(\leq N)$ and cancel like terms to obtain

$$
\begin{aligned}
& \left\|\xi_{h}\left(\cdot, t_{N_{1}}\right)\right\|_{L^{2}}^{2}+\varepsilon \Delta t \sum_{n=1}^{N_{1}}\left\|\xi_{h, x}\left(\cdot, t_{n}\right)\right\|_{L_{D}^{2}}^{2} \\
& \leq C \Delta t \sum_{n=0}^{N_{1}-1}\left\|\xi_{h}\left(\cdot, t_{n}\right)\right\|_{L^{2}}^{2}+C(\Delta t)^{2}\left(\varepsilon\left\|\frac{d u}{d t}\right\|_{L^{2}\left(0, T ; H^{1}\right)}^{2}+\|u\|_{L^{\infty}\left(0, T ; H^{1}\right)}^{2}\right. \\
& \left.\quad+\left\|\frac{d f}{d t}\right\|_{L^{2}\left(0, T ; L^{2}\right)}^{2}+\|f\|_{L^{2}\left(0, T ; L^{2}\right)}^{2}\right)+C\left(\min \left\{h^{2},(\Delta t)^{2}\right\}+h^{4}\right)\|u\|_{L^{\infty}\left(0, T ; H^{2}\right)}^{2} \\
& \quad+C \lambda h^{4}\left(\|u\|_{H^{1}\left(0, T ; H^{2}\right)}^{2}+\|u\|_{L^{\infty}\left(0, T ; H^{3}\right)}^{2}\right) .
\end{aligned}
$$

We then apply the Gronwall inequality to conclude

$$
\begin{aligned}
& \left\|\xi_{h}\right\|_{L_{\varepsilon}\left(0, T ; H_{D}^{1}(a, b)\right)} \\
& \leq C \Delta t\left(\sqrt{\varepsilon}\left\|\frac{d u}{d t}\right\|_{L^{2}\left(0, T ; H^{1}\right)}+\|u\|_{L^{\infty}\left(0, T ; H^{1}\right)}+\left\|\frac{d f}{d t}\right\|_{L^{2}\left(0, T ; L^{2}\right)}+\|f\|_{L^{2}\left(0, T ; L^{2}\right)}\right) \\
& \quad+C\left(\min \{h, \Delta t\}+h^{2}\right)\|u\|_{L^{\infty}\left(0, T ; H^{2}\right)}+C \lambda h^{2}\left(\|u\|_{H^{1}\left(0, T ; H^{2}\right)}+\|u\|_{L^{\infty}\left(0, T ; H^{3}\right)}\right) .
\end{aligned}
$$

The general constant $C$ depends exponentially on the final time $T$ in problem (2.1), due to the application of the Gronwall inequality, but does not depend on the parameter $\varepsilon$. We combine this estimate with (2.3) and the stability estimate of the true solution in Theorem 7.2 to finish the proof. 
4.2. The optimal-order error estimate for the MMOC and MMOCAA schemes. We prove an optimal-order error estimate for the MMOC scheme (3.19) and outline a similar estimate for the MMOCAA scheme.

Theorem 4.2. Assume $D, V \in L^{\infty}\left(0, T ; W_{\infty}^{4}(a, b)\right), f \in L^{2}\left(0, T ; H^{3}(a, b)\right)$, and $u_{o} \in H^{3}(a, b)$. Then the following optimal-order error estimate of the MMOC scheme holds uniformly with respect to $\varepsilon$ :

$$
\begin{aligned}
\left\|u_{h}-u\right\|_{L_{\varepsilon}\left(0, T ; H_{D}^{1}\right)} & \\
\leq & C \Delta t\left(\left\|u_{o}\right\|_{H_{\varepsilon}^{2}}+\varepsilon\left\|u_{o}\right\|_{H_{\varepsilon}^{3}}+\left\|\frac{d f}{d t}\right\|_{L^{2}\left(0, T ; L^{2}\right)}+\|f\|_{L^{2}\left(0, T ; H^{2}\right)}\right) \\
& +C\left(\min \{h, \Delta t\}+h^{2}\right)\left\|u_{o}\right\|_{H^{2}}+C \lambda h^{2}\left(\left\|u_{o}\right\|_{H^{3}}+\|f\|_{L^{2}\left(0, T ; H^{3}\right)}\right) .
\end{aligned}
$$

Here the constant $C$ is independent of $u$ and the parameter $\varepsilon$.

Proof. We let $e\left(x, t_{n}\right), \xi_{h}\left(x, t_{n}\right)$, and $\eta\left(x, t_{n}\right)$ be defined as in section 4.1 and choose $w_{h}(x)=\xi_{h}\left(x, t_{n}\right)$ in the MMOC reference equation (3.18) and the MMOC scheme (3.19). We subtract the latter from the former and rewrite the equation in terms of $\xi$ and $\eta$ as follows:

$$
\begin{aligned}
\int_{a}^{b} & \xi_{h}^{2}\left(x, t_{n}\right) d x+\varepsilon \Delta t \int_{a}^{b} D\left(x, t_{n}\right) \xi_{h, x}^{2}\left(x, t_{n}\right) d x+\Delta t \int_{a}^{b} V_{x}\left(x, t_{n}\right) \xi_{h}^{2}\left(x, t_{n}\right) d x \\
= & \int_{a}^{b} \xi_{h}\left(x_{h}^{*}, t_{n-1}\right) \xi_{h}\left(x, t_{n}\right) d x+\int_{a}^{b} \eta\left(x_{h}^{*}, t_{n-1}\right) \xi_{h}\left(x, t_{n}\right) d x \\
& -\int_{a}^{b} \eta\left(x, t_{n}\right) \xi_{h}\left(x, t_{n}\right) d x-\varepsilon \Delta t \int_{a}^{b} D\left(x, t_{n}\right) \eta_{x}\left(x, t_{n}\right) \xi_{h, x}\left(x, t_{n}\right) d x \\
& -\Delta t \int_{a}^{b} V_{x}\left(x, t_{n}\right) \eta\left(x, t_{n}\right) \xi_{h}\left(x, t_{n}\right) d x+E_{3}\left(u, \xi_{h}\right) .
\end{aligned}
$$

The first through fourth terms on the right-hand side of (4.13) were already bounded in (4.4)-(4.7). We need only to bound the remaining two terms on the right-hand side. The fifth term on the right-hand side is bounded by

$$
\begin{aligned}
\left|\Delta t \int_{a}^{b} V_{x}\left(x, t_{n}\right) \eta\left(x, t_{n}\right) \xi_{h}\left(x, t_{n}\right) d x\right| & \leq C \Delta t\left\|\xi_{h}\left(\cdot, t_{n}\right)\right\|_{L^{2}}\left\|\eta\left(\cdot, t_{n}\right)\right\|_{L^{2}} \\
& \leq C \Delta t\left\|\xi_{h}\left(\cdot, t_{n}\right)\right\|_{L^{2}}^{2}+C \Delta t h^{4}\|u\|_{L^{\infty}\left(0, T ; H^{2}\right)}^{2} .
\end{aligned}
$$

We use the expression of $E_{3}\left(u, \xi_{h}\right)$ (below (3.18)) to bound this term by

$$
\begin{aligned}
E_{3}\left(u, \xi_{h}\right) & \leq C(\Delta t)^{3 / 2}\left\|\xi_{h}\left(\cdot, t_{n}\right)\right\|_{L^{2}}\left\|\frac{d^{2} u}{d t^{2}}\right\|_{L^{2}\left(t_{n-1}, t_{n} ; L^{2}\right)} \\
& \leq C \Delta t\left\|\xi_{h}\left(\cdot, t_{n}\right)\right\|_{L^{2}}^{2}+C(\Delta t)^{2}\left\|\frac{d^{2} u}{d t^{2}}\right\|_{L^{2}\left(t_{n-1}, t_{n} ; L^{2}\right)}^{2}
\end{aligned}
$$

We combine these estimates and the estimates (4.4)-(4.7) to get

$$
\begin{aligned}
\left\|\xi_{h}\left(\cdot, t_{n}\right)\right\|_{L^{2}}^{2}+\varepsilon \Delta t\left\|\xi_{h, x}\left(\cdot, t_{n}\right)\right\|_{L_{D}^{2}}^{2} & \\
\leq & \frac{1+C \Delta t}{2}\left(\left\|\xi_{h}\left(\cdot, t_{n}\right)\right\|_{L^{2}}^{2}+\left\|\xi_{h}\left(\cdot, t_{n-1}\right)\right\|_{L^{2}}^{2}\right)+\frac{1}{4} \varepsilon \Delta t\left\|\xi_{h, x}\left(\cdot, t_{n}\right)\right\|_{L_{D}^{2}}^{2} \\
& +C(\Delta t)^{2}\left\|\frac{d^{2} u}{d t^{2}}\right\|_{L^{2}\left(t_{n-1}, t_{n} ; L^{2}\right)}^{2}+C \Delta t \min \left\{h^{2},(\Delta t)^{2}\right\}\|u\|_{L^{\infty}\left(0, T ; H^{2}\right)}^{2} \\
& +C h^{4}\left(\Delta t\|u\|_{L^{\infty}\left(0, T ; H^{2}\right)}^{2}+\lambda\left(\|u\|_{H^{1}\left(t_{n-1}, t_{n} ; H^{2}\right)}^{2}+\Delta t\|u\|_{L^{\infty}\left(0, T ; H^{3}\right)}^{2}\right)\right) .
\end{aligned}
$$

The rest of the proof is the same as that in Theorem 4.1. 
The MMOCAA scheme corrects the MMOC scheme by replacing $u_{h}\left(x_{h}^{*}, t_{n-1}\right)$ by $\check{u}_{h}\left(x_{h}^{*}, t_{n-1}\right)=u_{h}\left(x_{h}^{* *}, t_{n-1}\right)$, where $x_{h}^{* *}$ is an order $O\left((\Delta t)^{2}\right)$ perturbation to $x_{h}^{*}$. This does not affect the order of each term in the error analysis but introduces extra differences of the same term at $x_{h}^{*}$ and $x_{h}^{* *}$ and slightly complicates the analysis.

5. Error estimates for problem (2.1) with minimal or intermediate regularity. We prove a uniform stability estimate for the ELLAM, MMOC, and MMOCAA schemes, assuming minimal regularity of problem (2.1). We then use the theory of interpolation of operators to derive a priori error estimates, which hold uniformly with respect to $\varepsilon$, for problem (2.1) with minimal or intermediate regularity.

5.1. A uniform stability estimate. In this subsection we prove a uniform stability estimate for the ELLAM, MMOC, and MMOCAA schemes.

Theorem 5.1. Assume $V \in L^{\infty}\left(0, T ; W_{\infty}^{1}(a, b)\right), D \in L^{\infty}\left(0, T ; L^{\infty}(a, b)\right), u_{o} \in$ $L^{2}(a, b)$, and $f \in L^{2}\left(0, T ; L^{2}(a, b)\right)$. Let $u_{h}(x, 0)$ in the ELLAM scheme (3.13), the $M M O C$ scheme (3.19), or the MMOCAA scheme be the $L^{2}$ projection of $u_{o}(x)$. Then an uniform stability estimate holds:

$$
\left\|u_{h}\right\|_{L_{\varepsilon}\left(0, T ; H_{D}^{1}\right)} \leq C\left(\left\|u_{o}\right\|_{L^{2}}+\|f\|_{L^{2}\left(0, T ; L^{2}\right)}\right) .
$$

Proof. We choose $w_{h}\left(x, t_{n}\right)$ in the ELLAM scheme (3.13) to be $u_{h}\left(x, t_{n}\right)$ to get

$$
\begin{aligned}
& \int_{a}^{b} u_{h}^{2}\left(x, t_{n}\right) d x+\varepsilon \Delta t \int_{a}^{b} D\left(x, t_{n}\right) u_{h, x}^{2}\left(x, t_{n}\right) d x \\
& \quad=\int_{a}^{b} u_{h}\left(x_{h}^{*}, t_{n-1}\right) u_{h}\left(x, t_{n}\right) r_{h, x}\left(t_{n-1} ; x, t_{n}\right) d x+\Delta t \int_{a}^{b} f\left(x, t_{n}\right) u_{h}\left(x, t_{n}\right) d x .
\end{aligned}
$$

We bound the first term on the right-hand side similarly to estimate (4.4) and incorporate the estimate into (5.2). We cancel like terms and sum the resulting inequalities for $n=1, \ldots, N_{1}(\leq N)$ to get

$$
\begin{aligned}
& \left\|u_{h}\left(\cdot, t_{N_{1}}\right)\right\|_{L^{2}}^{2}+\varepsilon \sum_{n=1}^{N_{1}} \Delta t\left\|u_{h, x}\left(\cdot, t_{n}\right)\right\|_{L_{D}^{2}}^{2} \\
& \quad \leq C \Delta t \sum_{n=0}^{N_{1}}\left(\left\|u_{h}\left(\cdot, t_{n}\right)\right\|_{L^{2}}^{2}+\left\|f\left(\cdot, t_{n}\right)\right\|_{L^{2}\left(0, T ; L^{2}\right)}^{2}\right)+\left\|u_{o}\right\|_{L^{2}}^{2} .
\end{aligned}
$$

We choose $C \Delta t \leq 1 / 2$ and apply the Gronwall inequality to finish the proof of (5.1) in the context of the ELLAM scheme.

We similarly choose $w_{h}(x)=u_{h}\left(x, t_{n}\right)$ in the MMOC scheme (3.19) to get

$$
\begin{aligned}
\int_{a}^{b} & u_{h}^{2}\left(x, t_{n}\right) d x+\varepsilon \Delta t \int_{a}^{b} D\left(x, t_{n}\right) u_{h, x}^{2}\left(x, t_{n}\right) d x \\
= & \int_{a}^{b} u_{h}\left(x_{h}^{*}, t_{n-1}\right) u_{h}\left(x, t_{n}\right) r_{h, x}\left(t_{n-1} ; x, t_{n}\right) d x \\
& \quad-\Delta t \int_{a}^{b} V_{x}\left(x, t_{n}\right) u_{h}^{2}\left(x, t_{n}\right) d x+\Delta t \int_{a}^{b} f\left(x, t_{n}\right) u_{h}\left(x, t_{n}\right) d x .
\end{aligned}
$$

Compared with (5.2), the only extra term is the second term on the right-hand side of (5.3) that can be bounded by

$$
\left|\Delta t \int_{a}^{b} V_{x}\left(x, t_{n}\right) u_{h}^{2}\left(x, t_{n}\right) d x\right| \leq C \Delta t\left\|u_{h}\left(\cdot, t_{n}\right)\right\|_{L^{2}}^{2} .
$$


Thus, we can prove the stability estimate (5.1) for the MMOC as we did for the ELLAM. As for the MMOCAA, the only difference is the accumulation term at the time step $t_{n-1}$, in which the foot of the approximate characteristics is a perturbation of order $O\left((\Delta t)^{2}\right)$ to the Euler tracking. Therefore, the estimate (5.1) is still true.

5.2. Error estimates for problems with minimal or intermediate regularity. We apply the theory of interpolation of spaces to derive a priori error estimates for the ELLAM, MMOC, and MMOCAA schemes, which hold uniformly with respect to $\varepsilon$, for problem (2.1) with minimal or intermediate regularity.

THEOREM 5.2. Let $u$ be the true solution to problem (2.1) and $u_{h}$ be the numerical solution of the ELLAM scheme (3.13). Then the following error estimate holds uniformly with respect to $\varepsilon$ for $0<s<1$ :

$$
\begin{aligned}
\left\|u_{h}-u\right\|_{L_{\varepsilon}\left(0, T ; H_{D}^{1}\right)} \leq & C(\Delta t)^{s}\left(\left\|u_{o}\right\|_{B_{q}^{s}\left(L^{2}\right)}+\sqrt{\varepsilon}\left\|u_{o}\right\|_{B_{q}^{2 s}\left(L^{2}\right)}+\left\|\frac{d f}{d t}\right\|_{L^{2}\left(0, T ; L^{2}\right)}\right. \\
& \left.+\|f\|_{L^{2}\left(0, T ; B_{q}^{s}\left(L^{2}\right)\right)}\right)+C \min \left\{h^{s},(\Delta t)^{s}\right\}\left\|u_{o}\right\|_{B_{q}^{2 s}\left(L^{2}\right)} \\
& +C h^{2 s}\left(\left\|u_{o}\right\|_{B_{q}^{3 s}\left(L^{2}\right)}+\lambda\|f\|_{L^{2}\left(0, T ; B_{q}^{3 s}\left(L^{2}\right)\right)}\right) .
\end{aligned}
$$

Proof. We split $u_{h}-u=\left(u_{h}^{(1)}-u^{(1)}\right)+\left(u_{h}^{(2)}-u^{(2)}\right)$. Here $u_{h}^{(1)}$ and $u^{(1)}$ are the numerical and true solutions, respectively, to a homogeneous version of problem (2.1), whereas $u_{h}^{(2)}$ and $u^{(2)}$ are, respectively, the numerical and true solutions to problem (2.1) with zero initial data.

We combine the stability of the numerical solution $u_{h}^{(1)}$ and the true solution $u^{(1)}$, which is proved in Theorem 7.1, to obtain

$$
\left\|u_{h}^{(1)}-u^{(1)}\right\|_{L_{\varepsilon}\left(0, T ; H_{D}^{1}\right)} \leq C\left\|u_{o}\right\|_{L^{2}} .
$$

Theorem 4.1 gives an optimal-order error estimate assuming full regularity:

$$
\begin{aligned}
& \left\|u_{h}^{(1)}-u^{(1)}\right\|_{L_{\varepsilon}\left(0, T ; H_{D}^{1}\right)} \\
& \quad \leq C \Delta t\left\|u_{o}\right\|_{H_{\varepsilon}^{2}}+C\left(\min \{h, \Delta t\}+h^{2}\right)\left\|u_{o}\right\|_{H^{2}}+C \lambda h^{2}\left\|u_{o}\right\|_{H^{3}} .
\end{aligned}
$$

We use Lemma 2.1 with $m=3$ to interpolate $L^{2}(a, b)$ and the Sobolev space $H^{3}(a, b)$ :

$$
\left[L^{2}(a, b), H^{3}(a, b)\right]_{s, q}=B_{q}^{3 s}\left(L^{2}(a, b)\right), \quad 0<s<1 .
$$

Note that $\left[L^{2}(a, b), H^{3}(a, b)\right]_{k / 3,1} \subset H^{k}(a, b) \subset\left[L^{2}(a, b), H^{3}(a, b)\right]_{k / 3, \infty}$ for $k=1,2$. We apply Lemma 2.3 to reiterate the interpolation process on both ends to get

$\left[L^{2}(a, b), H^{k}(a, b)\right]_{s, q}=\left[L^{2}(a, b), H^{3}(a, b)\right]_{k s / 3, q}=B_{q}^{k s}\left(L^{2}(a, b)\right), 0<s<1, k=1,2$.

We apply Lemma 2.2 to the estimates (5.5) and (5.6) to conclude

$$
\begin{aligned}
& \left\|u_{h}^{(1)}-u^{(1)}\right\|_{L_{\varepsilon}\left(0, T ; H_{D}^{1}\right)} \\
& \leq C \lambda h^{2 s}\left\|u_{o}\right\|_{B_{q}^{3 s}\left(L^{2}\right)}+C\left(\min \left\{h^{s},(\Delta t)^{s}\right\}+h^{2 s}\right)\left\|u_{o}\right\|_{B_{q}^{2 s}\left(L^{2}\right)} \\
& \quad+C(\Delta t)^{s}\left(\left\|u_{o}\right\|_{B_{q}^{s}\left(L^{2}\right)}+\sqrt{\varepsilon}\left\|u_{o}\right\|_{B_{q}^{2 s}\left(L^{2}\right)}\right), \quad 0<s<1 .
\end{aligned}
$$

We use (5.1), (7.2), and (4.1) to bound $u_{h}^{(2)}-u^{(2)}$ by

$$
\left\|u_{h}^{(2)}-u^{(2)}\right\|_{L_{\varepsilon}\left(0, T ; H_{D}^{1}\right)} \leq C\|f\|_{L^{2}\left(0, T ; L^{2}\right)}
$$


and

$$
\begin{aligned}
\left\|u_{h}^{(2)}-u^{(2)}\right\|_{L_{\varepsilon}\left(0, T ; H_{D}^{1}\right)} \leq & C \Delta t\left(\left\|\frac{d f}{d t}\right\|_{L^{2}\left(0, T ; L^{2}\right)}+\|f\|_{L^{2}\left(0, T ; H^{1}\right)}\right) \\
& +C \lambda h^{2}\|f\|_{L^{2}\left(0, T ; H^{3}\right)} .
\end{aligned}
$$

We then use the interpolation to derive the following estimate:

$$
\begin{aligned}
\left\|u_{h}^{(2)}-u^{(2)}\right\|_{L_{\varepsilon}\left(0, T ; H_{D}^{1}\right)} \leq & C(\Delta t)^{s}\left(\left\|\frac{d f}{d t}\right\|_{L^{2}\left(0, T ; L^{2}\right)}+\|f\|_{L^{2}\left(0, T ; B_{q}^{s}\left(L^{2}\right)\right)}\right) \\
& +C \lambda h^{2 s}\|f\|_{L^{2}\left(0, T ; B_{q}^{3 s}\left(L^{2}\right)\right)} .
\end{aligned}
$$

We combine the estimates (5.7) and (5.10) to finish the proof of (5.4).

In the theorem, the coefficients are required to be in the appropriate interpolation spaces with proper fine tuning. An analogue can be proved for the MMOC and MMOCAA schemes in a similar manner.

THEOREM 5.3. Let $u$ be the true solution to problem (2.1) and $u_{h}$ be the numerical solution of the MMOC scheme (3.19) or the MMOCAA scheme. Then, for $0<s<1$, the following error estimate holds uniformly with respect to $\varepsilon$ :

$$
\begin{aligned}
\| u_{h} & -u \|_{L_{\varepsilon}\left(0, T ; H_{D}^{1}\right)} \\
\leq & C(\Delta t)^{s}\left(\left\|u_{o}\right\|_{B_{q}^{s}\left(L^{2}\right)}+\sqrt{\varepsilon}\left\|u_{o}\right\|_{B_{q}^{2 s}\left(L^{2}\right)}+\varepsilon^{3 / 2}\left\|u_{o}\right\|_{B_{q}^{3 s}\left(L^{2}\right)}\right. \\
& \left.+\left\|\frac{d f}{d t}\right\|_{L^{2}\left(0, T ; L^{2}\right)}+\|f\|_{L^{2}\left(0, T ; B_{q}^{2 s}\left(L^{2}\right)\right)}\right)+C \min \left\{h^{s},(\Delta t)^{s}\right\}\left\|u_{o}\right\|_{B_{q}^{2 s}\left(L^{2}\right)} \\
& +C h^{2 s}\left(\left\|u_{o}\right\|_{B_{q}^{3 s}\left(L^{2}\right)}+\lambda\|f\|_{L^{2}\left(0, T ; B_{q}^{3 s}\left(L^{2}\right)\right)}\right) .
\end{aligned}
$$

6. Auxiliary lemmas. We prove two auxiliary lemmas in this section. The first lemma addresses error bounds on the approximate characteristics to the true characteristics. The second lemma proves the optimal-order error bound in (4.6).

6.1. Estimates on approximations to characteristics. We prove several bounds on the differences between the approximate and true characteristics.

Lemma 6.1. Let $r\left(t ; x, t_{n}\right)$ and $r_{h}\left(t ; x, t_{n}\right)$ be the true and approximate characteristics defined in (3.3) and (3.12), respectively. Assume that $V, \frac{d V}{d t} \in L^{\infty}\left(0, T ; W_{\infty}^{1}(a, b)\right)$. Then the following estimates hold:

$$
\begin{gathered}
\left|x_{h}^{*}-x^{*}\right| \leq \frac{(\Delta t)^{2}}{2}\left\|\frac{d V}{d t}\right\|_{L^{\infty}\left(0, T ; L^{\infty}\right)}=O\left((\Delta t)^{2}\right), \\
\left|r_{x}\left(t ; x, t_{n}\right)-1\right| \leq \int_{t}^{t_{n}}\left|V_{x}\left(r\left(\theta ; x, t_{n}\right), \theta\right)\right| d \theta e^{\int_{t}^{t_{n}}\left|V_{x}\left(r\left(\theta ; x, t_{n}\right), \theta\right)\right| d \theta}=O\left(t_{n}-t\right),
\end{gathered}
$$

and

$$
\begin{aligned}
& \left|r_{h, x}\left(t_{n-1} ; x, t_{n}\right)-r_{x}\left(t_{n-1} ; x, t_{n}\right)\right| \\
& \quad \leq \frac{(\Delta t)^{2}}{2}\left(\left\|\frac{d V}{d t}\right\|_{L^{\infty}\left(0, T ; W_{\infty}^{1}\right)}+\|V\|_{L^{\infty}\left(0, T ; W_{\infty}^{1}\right)}^{2}\right) e^{\int_{t_{n-1}}^{t_{n}}\left|V_{x}\left(r\left(\theta ; x, t_{n}\right), \theta\right)\right| d \theta} \\
& \quad=O\left((\Delta t)^{2}\right) .
\end{aligned}
$$

Proof. The definition (3.3) directly yields

$$
r\left(t ; x, t_{n}\right)=x-\int_{t}^{t_{n}} V\left(r\left(\theta ; x, t_{n}\right), \theta\right) d \theta .
$$


We subtract this equation from (3.12) to get the following, which directly leads to the first inequality in (6.1):

$$
\begin{aligned}
\left|x_{h}^{*}-x^{*}\right| & =\left|\int_{t_{n-1}}^{t_{n}} V\left(r\left(t ; x, t_{n}\right), t\right) d t-V\left(x, t_{n}\right) \Delta t\right| \\
& =\left|\int_{t_{n-1}}^{t_{n}}\left[V\left(r\left(t ; x, t_{n}\right), t\right)-V\left(x, t_{n}\right)\right] d t\right| \\
& \leq \int_{t_{n-1}}^{t_{n}} \int_{\theta}^{t_{n}}\left|\frac{d V\left(r\left(\theta ; x, t_{n}\right), \theta\right)}{d \theta}\right| d \theta d t .
\end{aligned}
$$

Differentiating (6.3) leads to

$$
\begin{aligned}
r_{x}\left(t ; x, t_{n}\right)-1= & -\int_{t_{n}}^{t_{n}} V_{x}\left(r\left(\theta ; x, t_{n}\right), \theta\right) r_{x}\left(\theta ; x, t_{n}\right) d \theta \\
= & -\int_{t}^{t_{n}} V_{x}\left(r\left(\theta ; x, t_{n}\right), \theta\right) d \theta \\
& -\int_{t}^{t_{n}} V_{x}\left(r\left(\theta ; x, t_{n}\right), \theta\right)\left(r_{x}\left(\theta ; x, t_{n}\right)-1\right) d \theta
\end{aligned}
$$

Application of the Gronwall inequality leads to the second estimate in (6.1).

We differentiate (3.12) and use (6.4) to get

$$
\begin{aligned}
r_{h, x} & \left(t_{n-1} ; x, t_{n}\right)-r_{x}\left(t_{n-1} ; x, t_{n}\right) \\
= & \int_{t_{n-1}}^{t_{n}}\left[V_{x}\left(r\left(t ; x, t_{n}\right), t\right)-V_{x}\left(x, t_{n}\right)\right] d t \\
& -\int_{t_{n-1}}^{t_{n}} V_{x}\left(r\left(t ; x, t_{n}\right), t\right)\left[r_{h, x}\left(t ; x, t_{n}\right)-r_{x}\left(t ; x, t_{n}\right)+V_{x}\left(x, t_{n}\right)\left(t_{n}-t\right)\right] d t .
\end{aligned}
$$

Application of the Gronwall inequality to the following proves (6.2):

$$
\begin{aligned}
& \left|r_{h, x}\left(t_{n-1} ; x, t_{n}\right)-r_{x}\left(t_{n-1} ; x, t_{n}\right)\right| \\
& \quad \leq \frac{(\Delta t)^{2}}{2}\left(\left\|\frac{d V}{d t}\right\|_{L^{\infty}\left(0, T ; W_{\infty}^{1}\right)}+\|V\|_{L^{\infty}\left(0, T ; W_{\infty}^{1}\right)}^{2}\right) \\
& \quad+\int_{t_{n-1}}^{t_{n}}\left|V_{x}\left(r\left(t ; x, t_{n}\right), t\right)\right|\left|r_{h, x}\left(t ; x, t_{n}\right)-r_{x}\left(t ; x, t_{n}\right)\right| d t .
\end{aligned}
$$

6.2. A superconvergent estimate on interpolation. We prove the following superconvergence estimate on the interpolation error.

Lemma 6.2. Assume $u \in L^{\infty}\left(0, T ; H^{3}(a, b)\right) \cap H^{1}\left(0, T ; H^{2}(a, b)\right)$. Let $\Pi_{h} u \in$ $S_{h}(a, b)$ be the interpolation of $u$ and $\eta=\Pi_{h} u-u$. Let $\lambda$ be the parameter defined in Theorem 4.1. Then the following superconvergence estimate holds:

$$
\begin{aligned}
& \left|\int_{a}^{b} \eta\left(x_{h}^{*}, t_{n-1}\right) \xi_{h}\left(x, t_{n}\right) r_{h, x}\left(t_{n-1} ; x, t_{n}\right) d x-\int_{a}^{b} \eta\left(x, t_{n}\right) \xi_{h}\left(x, t_{n}\right) d x\right| \\
& \quad \leq C \Delta t\left\|\xi_{h}\left(\cdot, t_{n}\right)\right\|_{L^{2}}^{2}+C \Delta t\left(\min \left\{h^{2},(\Delta t)^{2}\right\}+h^{4}\right)\|u\|_{L^{\infty}\left(0, T ; H^{2}\right)}^{2} \\
& \quad+C(\Delta t)^{3}\|u\|_{L^{\infty}\left(0, T ; H^{1}\right)}^{2}+C \lambda h^{4}\left(\|u\|_{H^{1}\left(t_{n-1}, t_{n} ; H^{2}\right)}^{2}+\Delta t\|u\|_{L^{\infty}\left(0, T ; H^{3}\right)}^{2}\right) .
\end{aligned}
$$


Proof. We rewrite the left-hand side of (6.5) as

$$
\begin{aligned}
& \int_{a}^{b} \eta\left(x, t_{n}\right) \xi_{h}\left(x, t_{n}\right) d x-\int_{a}^{b} \eta\left(x_{h}^{*}, t_{n-1}\right) \xi_{h}\left(x, t_{n}\right) r_{h, x}\left(t_{n-1} ; x, t_{n}\right) d x \\
& \quad=\int_{a}^{b}\left(\eta\left(x, t_{n}\right)-\eta\left(x_{h}^{*}, t_{n-1}\right)\right) \xi_{h}\left(x, t_{n}\right) d x \\
& \quad+\Delta t \int_{a}^{b} \eta\left(x_{h}^{*}, t_{n-1}\right) \xi_{h}\left(x, t_{n}\right) V_{x}\left(x, t_{n}\right) d x .
\end{aligned}
$$

We bound the second term on the right-hand side in a similar way to (4.4):

$$
\begin{aligned}
& \left|\Delta t \int_{a}^{b} \eta\left(x_{h}^{*}, t_{n-1}\right) \xi_{h}\left(x, t_{n}\right) V_{x}\left(x, t_{n}\right) d x\right| \\
& \quad \leq C \Delta t\left\|\xi_{h}\left(\cdot, t_{n}\right)\right\|_{L^{2}}\left\|\eta\left(x_{h}^{*}, t_{n-1}\right)\right\|_{L^{2}(a, b)} \\
& \quad \leq C \Delta t\left\|\xi_{h}\left(\cdot, t_{n}\right)\right\|_{L^{2}}\left\|\eta\left(\cdot, t_{n-1}\right)\right\|_{L^{2}} \\
& \quad \leq C \Delta t\left\|\xi_{h}\left(\cdot, t_{n}\right)\right\|_{L^{2}}^{2}+C \Delta t h^{4}\|u\|_{L^{\infty}\left(0, T ; H^{2}\right)}^{2} .
\end{aligned}
$$

When $\mathrm{Cr} \geq 1$ that implies $h \leq C \Delta t$, we bound the first term in (6.6) by

$$
\begin{aligned}
& \left|\int_{a}^{b}\left(\eta\left(x, t_{n}\right)-\eta\left(x_{h}^{*}, t_{n-1}\right)\right) \xi_{h}\left(x, t_{n}\right) d x\right| \\
& \quad \leq C\left\|\xi_{h}\left(\cdot, t_{n}\right)\right\|_{L^{2}}\left(\left\|\eta\left(\cdot, t_{n}\right)\right\|_{L^{2}}+\left\|\eta\left(\cdot, t_{n-1}\right)\right\|_{L^{2}}\right) \\
& \quad \leq C h^{2}\left\|\xi_{h}\left(\cdot, t_{n}\right)\right\|_{L^{2}}\|u\|_{L^{\infty}\left(0, T ; H^{2}\right)} \\
& \quad \leq C \Delta t\left\|\xi_{h}\left(\cdot, t_{n}\right)\right\|_{L^{2}}^{2}+C \Delta t \min \left\{h^{2},(\Delta t)^{2}\right\}\|u\|_{L^{\infty}\left(0, T ; H^{2}\right)}^{2} .
\end{aligned}
$$

For $\mathrm{Cr}<1$, we decompose this term as follows:

$$
\begin{aligned}
& \int_{a}^{b}\left(\eta\left(x, t_{n}\right)-\eta\left(x_{h}^{*}, t_{n-1}\right)\right) \xi_{h}\left(x, t_{n}\right) d x \\
& =\int_{a}^{b} \int_{t_{n-1}}^{t_{n}} \eta_{t}(x, t) d t \xi_{h}\left(x, t_{n}\right) d x \\
& \quad+\int_{a}^{b}\left(\eta\left(x, t_{n-1}\right)-\eta\left(x_{h}^{*}, t_{n-1}\right)\right) \xi_{h}\left(x, t_{n}\right) d x
\end{aligned}
$$

The first term on the right-hand side is bounded by

$$
\begin{aligned}
& \left|\int_{a}^{b} \int_{t_{n-1}}^{t_{n}} \eta_{t}(x, t) d t \xi_{h}\left(x, t_{n}\right) d x\right| \\
& \quad \leq(\Delta t)^{1 / 2}\left\|\xi_{h}\left(\cdot, t_{n}\right)\right\|_{L^{2}}\|\eta\|_{H^{1}\left(t_{n-1}, t_{n} ; L^{2}\right)} \\
& \quad \leq \Delta t\left\|\xi_{h}\left(\cdot, t_{n}\right)\right\|_{L^{2}}^{2}+C h^{4}\|u\|_{H^{1}\left(t_{n-1}, t_{n} ; H^{2}\right)}^{2}
\end{aligned}
$$

We use the following expressions in the second term on the right side of (6.9):

$$
\begin{aligned}
\eta\left(x, t_{n-1}\right)-\eta\left(x_{h}^{*}, t_{n-1}\right) & =\int_{0}^{1} \frac{d}{d \theta} \eta\left(x_{h}^{*}+\theta\left(x-x_{h}^{*}\right), t_{n-1}\right) d \theta \\
& =\int_{0}^{1} \eta_{x}\left(x_{h}^{*}+\theta\left(x-x_{h}^{*}\right), t_{n-1}\right)\left(x-x_{h}^{*}\right) d \theta, \\
\frac{\partial}{\partial x}\left(\eta\left(x_{h}^{*}+\theta\left(x-x_{h}^{*}\right), t_{n-1}\right)\right) & =\eta_{x}\left(x_{h}^{*}+\theta\left(x-x_{h}^{*}\right), t_{n-1}\right)\left(x_{h, x}^{*}+\theta\left(1-x_{h, x}^{*}\right)\right) \\
& =\eta_{x}\left(x_{h}^{*}+\theta\left(x-x_{h}^{*}\right), t_{n-1}\right)\left(1-(1-\theta) V_{x}\left(x, t_{n}\right) \Delta t\right)
\end{aligned}
$$


and then integrate the resulting term by parts to yield

$$
\begin{aligned}
\int_{a}^{b} & \left(\eta\left(x, t_{n-1}\right)-\eta\left(x_{h}^{*}, t_{n-1}\right)\right) \xi_{h}\left(x, t_{n}\right) d x \\
= & \int_{a}^{b} \int_{0}^{1} \eta_{x}\left(x_{h}^{*}+\theta\left(x-x_{h}^{*}\right), t_{n-1}\right)\left(x-x_{h}^{*}\right) d \theta \xi_{h}\left(x, t_{n}\right) d x \\
= & \int_{a}^{b} \int_{0}^{1} \frac{\partial}{\partial x}\left(\eta\left(x_{h}^{*}+\theta\left(x-x_{h}^{*}\right), t_{n-1}\right)\right) \\
= & \int_{a}^{b} \int_{0}^{1} \frac{\partial}{\partial x}\left(\eta\left(x_{h}^{*}+\theta\left(x-x_{h}^{*}\right), t_{n-1}\right)\right)\left(x-x_{h}^{*}\right) d \theta \xi_{h}\left(x, t_{n}\right) d x \\
& +\int_{a}^{b} \int_{0}^{1} \frac{\partial}{\partial x}\left(\eta\left(x_{h}^{*}+\theta\left(x-x_{h}^{*}\right), t_{n-1}\right)\right) O\left((\Delta t)^{2}\right) d \theta \xi_{h}\left(x, t_{n}\right) d x \\
= & -\int_{0}^{1} \int_{a}^{b} \eta\left(x_{h}^{*}+\theta\left(x-x_{h}^{*}\right), t_{n-1}\right)\left(x-x_{h}^{*}\right)_{x} \xi_{h}\left(x, t_{n}\right) d x d \theta \\
& -\int_{0}^{1} \int_{a}^{b} \eta\left(x_{h}^{*}+\theta\left(x-x_{h}^{*}\right), t_{n-1}\right)\left(x-x_{h}^{*}\right) \xi_{h, x}\left(x, t_{n}\right) d x d \theta \\
& +\int_{a}^{b} \int_{0}^{1} \frac{\partial}{\partial x}\left(\eta\left(x_{h}^{*}+\theta\left(x-x_{h}^{*}\right), t_{n-1}\right)\right) O\left((\Delta t)^{2}\right) d \theta \xi_{h}\left(x, t_{n}\right) d x
\end{aligned}
$$

Let $y=x_{h}^{*}+\theta\left(x-x_{h}^{*}\right)$. We bound the first and third terms on the right side by

$$
\begin{aligned}
& \mid \int_{0}^{1} \int_{a}^{b} \eta\left(x_{h}^{*}+\theta\left(x-x_{h}^{*}\right), t_{n-1}\right)\left(x-x_{h}^{*}\right)_{x} \xi_{h}\left(x, t_{n}\right) d x d \theta \\
& \quad+\int_{a}^{b} \int_{0}^{1} \frac{\partial}{\partial x}\left(\eta\left(x_{h}^{*}+\theta\left(x-x_{h}^{*}\right), t_{n-1}\right)\right) O\left((\Delta t)^{2}\right) d \theta \xi_{h}\left(x, t_{n}\right) d x \mid \\
& \leq \Delta t \int_{0}^{1} \int_{a}^{b}\left|V_{x}\left(x, t_{n}\right)\left\|\eta\left(x_{h}^{*}+\theta\left(x-x_{h}^{*}\right), t_{n-1}\right)\right\| \xi_{h}\left(x, t_{n}\right)\right| d x d \theta \\
& \quad+C(\Delta t)^{2} \int_{0}^{1} \int_{a}^{b}\left|\eta_{x}\left(x_{h}^{*}+\theta\left(x-x_{h}^{*}\right), t_{n-1}\right)\right|\left|\xi_{h}\left(x, t_{n}\right)\right| d x d \theta \\
& \leq C \Delta t\left\|\xi_{h}\left(\cdot, t_{n}\right)\right\|_{L^{2}}\left[\left(\int_{0}^{1} \int_{a}^{b} \eta^{2}\left(y, t_{n-1}\right)\left(\theta+(1-\theta) x_{h, x}^{*}\right)^{-1} d y d \theta\right)^{1 / 2}\right. \\
& \left.\quad+\Delta t\left(\int_{0}^{1} \int_{a}^{b} \eta_{x}^{2}\left(y, t_{n-1}\right)\left(\theta+(1-\theta) x_{h, x}^{*}\right)^{-1} d y d \theta\right)^{1 / 2}\right] \\
& \leq C \Delta t\left\|\xi_{h}\left(\cdot, t_{n}\right)\right\|_{L^{2}}^{2}+C \Delta t h^{4}\|u\|_{L^{\infty}\left(0, T ; H^{2}\right)}^{2}+C(\Delta t)^{3}\|u\|_{L^{\infty}\left(0, T ; H^{1}\right)}^{2} .
\end{aligned}
$$

We decompose the second term on the right-hand side of (6.11) as

$$
\begin{aligned}
& \int_{0}^{1} \int_{a}^{b} \eta\left(x_{h}^{*}+\theta\left(x-x_{h}^{*}\right), t_{n-1}\right)\left(x-x_{h}^{*}\right) \xi_{h, x}\left(x, t_{n}\right) d x d \theta \\
& =\Delta t \int_{a}^{b} V\left(x, t_{n}\right) \xi_{h, x}\left(x, t_{n}\right)\left(\eta\left(x, t_{n-1}\right)\right. \\
& \left.\quad+\int_{0}^{1} \int_{0}^{1} \frac{d}{d \gamma} \eta\left(x+\gamma(1-\theta)\left(x_{h}^{*}-x\right), t_{n-1}\right) d \gamma d \theta\right) d x \\
& =\Delta t \int_{a}^{b} V\left(x, t_{n}\right) \xi_{h, x}\left(x, t_{n}\right) \eta\left(x, t_{n-1}\right) d x \\
& +\Delta t \int_{0}^{1} \int_{0}^{1} \int_{a}^{b} V\left(x, t_{n}\right) \xi_{h, x}\left(x, t_{n}\right)(1-\theta)\left(x_{h}^{*}-x\right) \\
& \quad \times \eta_{x}\left(x+\gamma(1-\theta)\left(x_{h}^{*}-x\right), t_{n-1}\right) d x d \gamma d \theta .
\end{aligned}
$$


We use the inverse inequality (2.3) to bound the second term by

$$
\begin{aligned}
& \mid \Delta t \int_{0}^{1} \int_{0}^{1} \int_{a}^{b} V\left(x, t_{n}\right) \xi_{h, x}\left(x, t_{n}\right)(1-\theta)\left(x_{h}^{*}-x\right) \\
& \quad \times \eta_{x}\left(x+\gamma(1-\theta)\left(x_{h}^{*}-x\right), t_{n-1}\right) d x d \gamma d \theta \mid \\
& \leq C(\Delta t)^{2} h\left\|\xi_{h, x}\left(\cdot, t_{n}\right)\right\|_{L^{2}}\left\|u\left(\cdot, t_{n-1}\right)\right\|_{H^{2}} \\
& \leq C \Delta t\left\|\xi_{h}\left(\cdot, t_{n}\right)\right\|_{L^{2}}^{2}+C(\Delta t)^{3}\|u\|_{L^{\infty}\left(0, T ; H^{2}\right)}^{2} .
\end{aligned}
$$

A standard estimate of the first term on the right-hand side of (6.13) yields

$$
\begin{aligned}
\left|\Delta t \int_{a}^{b} V\left(x, t_{n}\right) \eta\left(x, t_{n-1}\right) \xi_{h, x}\left(x, t_{n}\right) d x\right| & \leq C \Delta t\left\|\xi_{h, x}\left(\cdot, t_{n}\right)\right\|_{L^{2}}\left\|\eta\left(\cdot, t_{n-1}\right)\right\|_{L^{2}} \\
& \leq C \Delta t h^{2}\left\|\xi_{h, x}\left(\cdot, t_{n}\right)\right\|_{L^{2}}\left\|u\left(\cdot, t_{n-1}\right)\right\|_{H^{2}} \\
& \leq C \Delta t\left\|\xi_{h}\left(\cdot, t_{n}\right)\right\|_{L^{2}}^{2}+C \Delta t h^{2}\left\|u\left(\cdot, t_{n-1}\right)\right\|_{H^{2}} .
\end{aligned}
$$

This will result in a suboptimal-order estimate of order $O(h+\Delta t)$ for the ELLAM scheme. To derive an optimal-order estimate, we sum this term by parts to obtain

$$
\begin{aligned}
\Delta t & \int_{a}^{b} V\left(x, t_{n}\right) \eta\left(x, t_{n-1}\right) \xi_{h, x}\left(x, t_{n}\right) d x \\
= & -\frac{\Delta t}{h} \sum_{i=1}^{I} \int_{x_{i-1}}^{x_{i}}\left(V\left(x+h, t_{n}\right)-V\left(x, t_{n}\right)\right) \eta\left(x, t_{n-1}\right) \xi_{h}\left(x_{i}, t_{n}\right) d x \\
& \quad-\frac{\Delta t}{h} \sum_{i=1}^{I} \int_{x_{i-1}}^{x_{i}}\left(\eta\left(x+h, t_{n-1}\right)-\eta\left(x, t_{n-1}\right)\right) V\left(x+h, t_{n}\right) \xi_{h}\left(x_{i}, t_{n}\right) d x .
\end{aligned}
$$

We bound the first term on the right-hand side of (6.15) by

$$
\begin{aligned}
& \left|\frac{\Delta t}{h} \sum_{i=1}^{I} \int_{x_{i-1}}^{x_{i}}\left(V\left(x+h, t_{n}\right)-V\left(x, t_{n}\right)\right) \eta\left(x, t_{n-1}\right) \xi_{h}\left(x_{i}, t_{n}\right) d x\right| \\
& \quad \leq C \Delta t\left\|\xi_{h}\left(\cdot, t_{n}\right)\right\|_{L^{2}}\left\|\eta\left(\cdot, t_{n-1}\right)\right\|_{L^{2}} \\
& \quad \leq C \Delta t\left\|\xi_{h}\left(\cdot, t_{n}\right)\right\|_{L^{2}}^{2}+C \Delta t h^{4}\|u\|_{L^{\infty}\left(0, T ; H^{2}\right)}^{2},
\end{aligned}
$$

where we have used the equivalence between the discrete and continuous $L^{2}$ norms.

However, if we similarly bound the second term on the right side of (6.15), we can obtain only a suboptimal-order estimate. To derive an optimal-order estimate, we introduce an auxiliary function $\psi(x, t)$ by

$$
\psi(x, t)=u(x+h, t)-u(x, t)=\int_{0}^{h} u_{\alpha}(\alpha+x, t) d \alpha .
$$

Because $\eta\left(x+h, t_{n-1}\right)$ is a shift of $\eta\left(x, t_{n-1}\right)$ by one grid point, so the forward difference operator and the shift operator are commutative:

$$
\begin{aligned}
\eta\left(x+h, t_{n-1}\right)-\eta\left(x, t_{n-1}\right) & =\left(\Pi_{h}-\mathrm{I}\right) u\left(x+h, t_{n-1}\right)-\left(\Pi_{h}-\mathrm{I}\right) u\left(x, t_{n-1}\right) \\
& =\left(\Pi_{h}-\mathrm{I}\right)\left(u\left(x+h, t_{n-1}\right)-u\left(x, t_{n-1}\right)\right) \\
& =\left(\Pi_{h}-\mathrm{I}\right) \psi\left(x, t_{n-1}\right) .
\end{aligned}
$$


Inserting this identity into the second term on the right-hand side of (6.15) gives

$$
\begin{aligned}
& \left|\frac{\Delta t}{h} \sum_{i=1}^{I} \int_{x_{i-1}}^{x_{i}}\left(\eta\left(x+h, t_{n}\right)-\eta\left(x, t_{n}\right)\right) V\left(x+h, t_{n}\right) \xi_{h}\left(x_{i}, t_{n}\right) d x\right| \\
& \quad \leq \frac{C \Delta t}{h}\left\|\xi_{h}\left(\cdot, t_{n}\right)\right\|_{L^{2}}\left\|\left(\Pi_{h}-\mathrm{I}\right) \psi\left(\cdot, t_{n-1}\right)\right\|_{L^{2}} \leq C \Delta t h\left\|\xi_{h}\left(\cdot, t_{n}\right)\right\|_{L^{2}}\left\|\psi\left(\cdot, t_{n-1}\right)\right\|_{H^{2}} \\
& \quad \leq C \Delta t\left\|\xi_{h}\left(\cdot, t_{n}\right)\right\|_{L^{2}}^{2}+C \Delta t h^{4}\|u\|_{L^{\infty}\left(0, T ; H^{3}\right)}^{2} .
\end{aligned}
$$

Combining all of these estimates, we have proved (6.5).

7. Uniform stability estimates of the exact solutions. The existence, uniqueness, and stability estimates for problem (2.1) can be found, e.g., in [9]. We derive a priori stability estimates for problem (2.1) in $\varepsilon$-weighted norms, which hold uniformly with respect to $\varepsilon$, under different regularity assumptions.

7.1. A generic stability estimate with minimal regularity assumption. We prove a priori stability estimates for a slightly more general initial-boundary value problem than problem (2.1) with minimal regularity assumption:

$$
\begin{aligned}
z_{t}+\left(\bar{V}(x, t) z-\varepsilon \bar{D}(x, t) z_{x}\right)_{x}+\bar{R}(x, t) z & =\bar{f}(x, t), \quad(x, t) \in(a, b) \times(0, T], \\
z(x, 0) & =z_{o}(x),
\end{aligned}
$$

which is closed by a periodic boundary condition at $x=a$ and $x=b$.

Theorem 7.1. Assume $\bar{D} \in L^{\infty}\left(0, T ; L^{\infty}\right), \bar{R} \in L^{1}\left(0, T ; L^{\infty}\right), \bar{V} \in L^{1}\left(0, T ; W_{\infty}^{1}\right)$, $z_{o} \in L^{2}(a, b)$, and $\bar{f} \in L^{2}\left(0, T ; L^{2}\right)$. Then the following estimate holds:

$$
\begin{aligned}
\|z\|_{L_{\varepsilon}\left(0, T ; H_{D}^{1}\right)} & \leq 2\left(\left\|z_{o}\right\|_{L^{2}}+\|\bar{f}\|_{L^{2}\left(0, T ; L^{2}\right)}\right) e^{\frac{1}{2}\left\|\left(1-2 \bar{R}-\bar{V}_{x}\right)^{+}\right\|_{L^{1}\left(0, T ; L^{\infty}\right)}} \\
& \leq C\left(\left\|z_{o}\right\|_{L^{2}}+\|\bar{f}\|_{L^{2}\left(0, T ; L^{2}\right)}\right) .
\end{aligned}
$$

Here $C$ depends on $\left\|\left(1-2 \bar{R}-\bar{V}_{x}\right)^{+}\right\|_{L^{1}\left(0, T ; L^{\infty}\right)}$ but not on $\varepsilon, g^{+}(x, t)=\max \{g(x, t), 0\}$.

Proof. We combine $z_{t}+\bar{V} z_{x}$ in (7.1) to form a material derivative $\frac{d z}{d t}$ along the characteristics $x=r(t ; \bar{x}, \bar{t})$ defined by (3.3) with $V$ being replaced by $\bar{V}$. We multiply the equation by $z(x, t)$ and integrate the resulting equation to get

$$
\int_{a}^{b} \frac{d z}{d t} z d x+\varepsilon \int_{a}^{b} \bar{D}(x, t) z_{x}^{2} d x+\int_{a}^{b}\left(\bar{R}(x, t)+\bar{V}_{x}(x, t)\right) z^{2} d x=\int_{a}^{b} \bar{f}(x, t) z d x
$$

We apply the Reynolds transport theorem

$$
\frac{d}{d t} \int_{r(t ; a, \bar{t})}^{r(t ; b, \bar{t})} g d x=\int_{r(t ; a, \bar{t})}^{r(t ; b, \bar{t})}\left(\frac{d g}{d t}+\bar{V}_{x} g\right) d x
$$

to $g=z^{2}(x, t)$ at $t=\bar{t}$ to rewrite the weak formulation as

$$
\frac{1}{2} \frac{d}{d t} \int_{a}^{b} z^{2} d x+\varepsilon \int_{a}^{b} \bar{D} z_{x}^{2} d x+\int_{a}^{b}\left(\bar{R}+\frac{1}{2} \bar{V}_{x}\right) z^{2} d x=\int_{a}^{b} f z d x .
$$


Integrating this equation from $t=0$ to $t=\bar{t}$ yields

$$
\begin{aligned}
\int_{a}^{b} & z^{2}(x, \bar{t}) d x+2 \varepsilon \int_{0}^{\bar{t}} \int_{a}^{b} \bar{D}(x, t) z_{x}^{2}(x, t) d x d t \\
= & \int_{a}^{b} z^{2}(x, \bar{t}) d x+2 \varepsilon \int_{0}^{\bar{t}} \int_{r(t ; a, \bar{t})}^{r(t ; b, \bar{t})} \bar{D}(x, t) z_{x}^{2}(x, t) d x d t \\
\leq & \int_{r(0 ; a, \bar{t})}^{r(0 ; b, \bar{t})} z_{o}^{2}(x) d x+\int_{0}^{\bar{t}} \int_{r(t ; a, \bar{t})}^{r(t ; b, \bar{t})} \bar{f}^{2}(x, t) d x d t \\
& +\int_{0}^{t}\left\|\left(1-2 \bar{R}-\bar{V}_{x}\right)^{+}(\cdot, t)\right\|_{L^{\infty}(a, b)} \int_{r(t ; ; ;, \bar{t})}^{r(t, \bar{t})} z^{2}(x, t) d x d t \\
= & \left\|z_{o}\right\|_{L^{2}}^{2}+\int_{0}^{\bar{t}}\|\bar{f}(\cdot, t)\|_{L^{2}}^{2} d t+\int_{0}^{\bar{t}}\left\|\left(1-2 \bar{R}-\bar{V}_{x}\right)^{+}(\cdot, t)\right\|_{L^{\infty}(a, b)}\|z(\cdot, t)\|_{L^{2}}^{2} d t
\end{aligned}
$$

Here we have used the fact that $\int_{r(t ; a, \bar{t})}^{r(t ; b, \bar{t})} g(x) d x=\int_{a}^{b} g(x) d x$. Applying the Gronwall inequality finishes the proof of (7.2).

7.2. Uniform stability estimates for problem (2.1). In this subsection we prove a priori stability estimates for problem (2.1) in different Sobolev norms.

TheOrem 7.2. Assume $D, V \in L^{\infty}\left(0, T ; W_{\infty}^{k+1}(a, b)\right), u_{o} \in H^{k}(a, b)$, and $f \in$ $L^{2}\left(0, T ; H^{k}(a, b)\right)$. Then the following stability estimate holds for problem (2.1):

$$
\|u\|_{L_{\varepsilon}\left(0, T ; H^{k+1}\right)} \leq C\left(\left\|u_{o}\right\|_{H^{k}}+\|f\|_{L^{2}\left(0, T ; H^{k}\right)}\right),
$$

where $C=C\left(\|D\|_{L^{\infty}\left(0, T ; W_{\infty}^{k+1}\right)},\|V\|_{L^{\infty}\left(0, T ; W_{\infty}^{k+1}\right)}\right)$, but not on $\varepsilon$. Further, if $D, V \in$ $L^{\infty}\left(0, T ; W_{\infty}^{4}(a, b)\right), u_{o} \in H^{3}(a, b)$, and $f \in L^{2}\left(0, T ; H^{3}(a, b)\right)$, we have

$$
\|u\|_{H^{1}\left(0, T ; H^{2}\right)} \leq C\left(\left\|u_{o}\right\|_{H^{3}}+\|f\|_{L^{2}\left(0, T ; H^{3}\right)}\right)
$$

and

$$
\left\|\frac{d^{2} u}{d t^{2}}\right\|_{L^{2}\left(0, T ; L^{2}\right)} \leq C\left(\varepsilon\left\|u_{o}\right\|_{H_{\varepsilon}^{3}}+\left\|u_{o}\right\|_{H_{\varepsilon}^{1}}+\|f\|_{L^{2}\left(0, T ; H^{2}\right)}+\left\|\frac{d f}{d t}\right\|_{L^{2}\left(0, T ; L^{2}\right)}\right) .
$$

Here $C=C\left(\|D\|_{L^{\infty}\left(0, T ; W_{\infty}^{4}\right)},\|V\|_{L^{\infty}\left(0, T ; W_{\infty}^{4}\right)}\right)$, but not on $\varepsilon$. Finally, assume $D, V \in$ $L^{\infty}\left(0, T ; W_{\infty}^{l+1}(a, b)\right), u_{o} \in H^{l}(a, b)$, and $f \in L^{2}\left(0, T ; H^{l}(a, b)\right)$ for $l=0,1$. We have

$$
\left\|\frac{d u}{d t}\right\|_{L^{2}\left(0, T ; H^{l}\right)} \leq C\left(\left\|u_{o}\right\|_{H_{\varepsilon}^{l+1}}+\|f\|_{L^{2}\left(0, T ; H^{l}\right)}\right) .
$$

Proof. (7.3) for $k=0$ is a direct consequence of (7.2). To prove (7.3) for $k=1$, we differentiate problem (2.1) with respect to $x$. The resulting equation corresponds to (7.1) with $z=u_{x}, \bar{D}=D, \bar{V}=V-\varepsilon D_{x}, \bar{R}=V_{x}$, and $\bar{f}=f_{x}-V_{x x}$. The estimate (7.2) yields (7.3) with $k=1$. We prove (7.3) for $k \geq 2$ by induction.

To prove the estimate (7.4) we use the governing equation in (2.1) to express $u_{t}$ in terms of spatial derivatives and bound these spatial derivatives by the estimate (7.3). We similarly bound $\frac{d u}{d t}$ in terms of spatial derivatives as follows:

$$
\left\|\frac{d u}{d t}\right\|_{L^{2}\left(0, T ; H^{l}\right)} \leq C\left(\varepsilon\left\|\left(D u_{x}\right)_{x}\right\|_{L^{2}\left(0, T ; H^{l}\right)}+\left\|V_{x} u\right\|_{L^{2}\left(0, T ; H^{l}\right)}+\|f\|_{L^{2}\left(0, T ; H^{l}\right)}\right) .
$$


This together with (7.3) proves the estimate of $\frac{d u}{d t}$. We similarly express $\frac{d^{2} u}{d t^{2}}$ as

$$
\begin{aligned}
& \left\|\frac{d^{2} u}{d t^{2}}\right\|_{L^{2}\left(0, T ; L^{2}\right)} \\
& =\left\|\varepsilon\left[D\left(\varepsilon\left(D u_{x}\right)_{x}-V_{x} u+f\right)_{x}\right]_{x}+\varepsilon\left(\frac{d D}{d t} u_{x}\right)_{x}-V_{x} \frac{d u}{d t}-\frac{d V_{x}}{d t} u+\frac{d f}{d t}\right\|_{L^{2}\left(0, T ; L^{2}\right)} \\
& \leq \varepsilon^{2}\|u\|_{L^{2}\left(0, T ; H^{4}\right)}+\varepsilon\|u\|_{L^{2}\left(0, T ; H^{2}\right)}+\|u\|_{L^{2}\left(0, T ; L^{2}\right)}+\|f\|_{L^{2}\left(0, T ; H^{2}\right)} \\
& \quad+\left\|\frac{d u}{d t}\right\|_{L^{2}\left(0, T ; L^{2}\right)}+\left\|\frac{d f}{d t}\right\|_{L^{2}\left(0, T ; L^{2}\right)} .
\end{aligned}
$$

We combine this inequality with estimates (7.3) and (7.6) to finish the proof.

8. Concluding remarks. In this section we summarize the main results in this paper and address several related issues. We also carry out numerical example runs to verify the theoretical estimates numerically. We conclude the paper by briefly discussing the directions of future work.

8.1. The $\varepsilon$-weighted energy norm and $\boldsymbol{L}^{\infty}$ norm. In the context of stationary convection-diffusion equations, the location of internal and boundary layers is known a priori. A piecewise-uniform mesh was proposed and analyzed by Shishkin to resolve the boundary and internal layers. Moreover, an $\varepsilon$-uniform $L^{\infty}$ error estimate was proved for numerical methods with Shishkin mesh [11, 14]. However, in the context of transient convection-diffusion equations, the fronts are dynamic and do not always coincide with the spatial mesh. Thus, although an $\varepsilon$-uniform error estimate in the $L^{\infty}$ norm is ideal, it is generally impossible especially in the context of multiple space dimensions and in the limiting case of $\varepsilon=0$. This is why the $L^{\infty}$ norm is not used in the numerical methods for hyperbolic conservation laws [13].

In this paper we derived $\varepsilon$-uniform error estimates in the $\varepsilon$-weighted energy norm $\|\cdot\|_{H_{\varepsilon}^{1}}$. We now discuss the relation between the error estimates measured in $\|\cdot\|_{L^{\infty}}$ and in $\|\cdot\|_{H_{\varepsilon}^{1}}$. In the context of an exponential layer (say, located at $x=1$ ), the approximation error $e$ is expected to be of the form $[11,14]$

$$
e=\exp \left(\frac{-(1-x)}{\varepsilon}\right), \quad 0 \leq x \leq 1
$$

$\|e\|_{L^{\infty}}=O(1)$ and $\|e\|_{H_{\varepsilon}^{1}(0,1)}=\|e\|_{L^{2}(0,1)}+\sqrt{\varepsilon}\left\|e_{x}\right\|_{L^{2}(0,1)}=\sqrt{\varepsilon}+\sqrt{\varepsilon} \cdot(1 / \sqrt{\varepsilon})=O(1)$. Thus, $\|e\|_{L^{\infty}}$ is comparable to $\|e\|_{H_{\varepsilon}^{1}(0,1)}$, and both norms recognize the exponential layer. When problem (2.1) has a smooth solution, $\|e\|_{L^{\infty}}=O\left(h^{2}\right)$ and $\|e\|_{H_{\varepsilon}^{1}(0,1)}=$ $\|e\|_{L^{2}(0,1)}+\sqrt{\varepsilon}\left\|e_{x}\right\|_{L^{2}(0,1)}=O\left(h^{2}+\sqrt{\varepsilon} h\right)=O\left(h^{2}\right)$ for $\varepsilon<h^{2}$. Thus, $\|e\|_{L^{\infty}}$ is still comparable to $\|e\|_{H_{\varepsilon}^{1}(0,1)}$.

In the context of a parabolic layer (say, located at $x=1$ ), the approximation error $e$ is expected to be of the form $[11,14]$

$$
e=\exp \left(\frac{-(1-x)}{\sqrt{\varepsilon}}\right), \quad 0 \leq x \leq 1
$$

For a parabolic layer, $\|e\|_{L^{\infty}}=O(1)$ and $\|e\|_{H_{\varepsilon}^{1}(0,1)}=\|e\|_{L^{2}(0,1)}+\sqrt{\varepsilon}\left\|e_{x}\right\|_{L^{2}(0,1)}=$ $\varepsilon^{1 / 4}+\sqrt{\varepsilon} \cdot \varepsilon^{-1 / 4}=O\left(\varepsilon^{1 / 4}\right)$. In this case, the $L^{\infty}$ norm still recognizes the parabolic layer, but the $\varepsilon$-weighted norm does not recognize the layer.

In summary, the $\varepsilon$-weighted norm is comparable to the $L^{\infty}$ norm in the cases of a smooth solution or an exponential layer. An exponential layer exhibits the strongest layer behavior and is of the major concern from a numerical and analysis viewpoint. 
On the other hand, in the context of a parabolic layer, $\|\cdot\|_{H_{\varepsilon}^{1}}$ is somewhat weaker than $\|\cdot\|_{L^{\infty}}$. Thus, the $\varepsilon$-weighted norm is the most feasible measure for transient convection-diffusion equations and is closely related to the $L^{\infty}$ norm. The $L^{\infty}$ norm is an ideal but impossible measure in this context.

8.2. Measurements in Besov spaces. In Theorems 5.2 and 5.3, we used the interpolation theory and stability estimates to derive an $\varepsilon$-uniform estimate for problem (2.1) with minimal or intermediate regularity, where the initial and right-hand side data were measured in Besov spaces that generate a refined scale of smoothness and convergence rate. As an example, we consider an initial configuration that contains interior layers. Note that the indicator function $\chi_{(0.4,0.8)}$ (introduced before estimate (4.11)) satisfies $\chi_{(0.4,0.8)} \in H^{1 / 2-\delta}(0,1)$ for any $0<\delta \leq 1 / 2$ but $\notin H^{1 / 2}(0,1)$. Direct calculation shows that

$$
\left\|\chi_{(0.4,0.8)}\right\|_{H^{1 / 2-\delta}(0,1)}=O\left(\delta^{-1 / 2}\right) \rightarrow \infty \quad \text { as } \delta \rightarrow 0 .
$$

Consequently, the convergence rate in the estimates (5.4) and (5.11) will be $s=$ $1 / 4-\delta / 2$ in the case of $\mathrm{Cr} \geq 1$, and the norm will blow up as $\delta \rightarrow 0$, if the initial configuration is measured in the Sobolev spaces. On the other hand, it can be verified that $\chi_{(0.4,0.8)} \in B_{\infty}^{1 / 2}\left(L^{2}(0,1)\right)$ but $\notin B_{q}^{1 / 2}\left(L^{2}(0,1)\right)$ for $q<\infty$. With this measure of the same initial data, the estimates (5.4) and (5.11) yield a sharp convergence rate of order $s=1 / 4$ in the case of $\mathrm{Cr} \geq 1$.

8.3. Numerical experiments. Various numerical experiments were reported in the literature, which confirmed spatial and temporal convergence rates of EulerianLagrangian methods (see, e.g., $[7,15,18]$ ). In this section we conduct numerical experiments to observe the convergence behavior of the truncation error $u_{h}-u$ and its dependence on $\varepsilon$. We simulate the transport of a Gaussian pulse subject to (2.1) with the initial configuration being given by

$$
u_{o}(x)=\exp \left(-\frac{\left(x-x_{c}\right)^{2}}{2 \sigma^{2}}\right)
$$

where $x_{c}$ and $\sigma$ are the centered and standard deviations, respectively, of the Gaussian pulse. The analytical solution $u(x, t)$ for a homogeneous equation (2.1) is given by

$$
u(x, t)=\frac{\sqrt{2} \sigma}{\sqrt{2 \sigma^{2}+4 \varepsilon t}} \exp \left(-\frac{\left(x-x_{c}-V t\right)^{2}}{2 \sigma^{2}+4 \varepsilon t}\right) .
$$

In the numerical example runs, the spatial domain is $(a, b)=(0,3)$, and the time interval is $(0, T)=(0,1)$. We select $V=1$ and $D=1$ so the convection dominance is controlled by the magnitude of $\varepsilon$, which is chosen to be $0.001,0.0001$, and 0 . We also choose $x_{c}=0.5$, and $\sigma=0.1$. We fix a small time step $\Delta t$ and use a linear regression to fit the convergence rates and the associated constants in the weighted energy norm

$$
\left\|u_{h}-u\right\| \leq C_{\alpha} h^{\alpha}
$$

The results are presented in Table 8.1, which shows that the ELLAM scheme maintains second-order accuracy in space. Moreover, these convergence rates hold uniformly as $\varepsilon$ tends to zero, even in the limiting case of $\varepsilon=0$, as predicted by the theorems proved in this paper. 
TABLE 8.1

Spatial convergence rates in the $\varepsilon$-weighted energy norm with $\Delta t=1 / 100$.

\begin{tabular}{|c|c|c|c|}
\hline$h$ & $\varepsilon=0.001$ & $\varepsilon=0.0001$ & $\varepsilon=0$ \\
\hline $1 / 10$ & $5.74 \times 10^{-2}$ & $7.52 \times 10^{-2}$ & $7.76 \times 10^{-2}$ \\
\hline $1 / 20$ & $7.99 \times 10^{-3}$ & $1.02 \times 10^{-2}$ & $1.05 \times 10^{-2}$ \\
\hline $1 / 30$ & $2.68 \times 10^{-3}$ & $3.10 \times 10^{-3}$ & $3.15 \times 10^{-3}$ \\
\hline $1 / 40$ & $1.24 \times 10^{-3}$ & $1.36 \times 10^{-3}$ & $1.37 \times 10^{-3}$ \\
\hline $1 / 60$ & $4.01 \times 10^{-4}$ & $4.31 \times 10^{-4}$ & $4.32 \times 10^{-4}$ \\
\hline $1 / 80$ & $1.86 \times 10^{-4}$ & $2.12 \times 10^{-4}$ & $2.15 \times 10^{-4}$ \\
\hline & $C_{\alpha}=32, \alpha=2.75$ & $C_{\alpha}=51, \alpha=2.84$ & $C_{\alpha}=54, \alpha=2.86$ \\
\hline
\end{tabular}

8.4. Summary and discussions. In this paper we proved $\varepsilon$-uniform error estimates in the $\varepsilon$-weighted energy norm for the ELLAM, MMOC, and MMOCAA schemes for one-dimensional singularly perturbed, time-dependent convection-diffusion equations with periodic boundary conditions. The estimates were derived on a uniform space-time partition with no upstream weighting or local grid refinement or any other special arrangements of the grid, so these estimates justify the strength of Eulerian-Lagrangian methods. The analysis fully utilizes the simplicity of the one space dimension and the periodic boundary conditions.

However, a multidimensional analogue of problem (2.1) with general boundary conditions presents much more severe challenges, due to the complication of multiple space dimensions, the solution structures, and the appearance of boundary and interior layers. These issues will be investigated in the near future.

Acknowledgments. This work was done during the visit of the first author to Shandong University. The authors express their sincere thanks to the referees for their very helpful comments and suggestions, which greatly improved the quality of this paper.

\section{REFERENCES}

[1] T. Arbogast And M.F. Wheeler, A characteristics-mixed finite element method for advection-dominated transport problems, SIAM J. Numer. Anal., 32 (1995), pp. 404-424.

[2] C. Bennett and R.C. Sharpley, Interpolation of Operators, Academic, New York, 1988.

[3] M.A. Celia, T.F. Russell, I. Herrera, and R.E. Ewing, An Eulerian-Lagrangian localized adjoint method for the advection-diffusion equation, Adv. Water Resources, 13 (1990), pp. $187-206$.

[4] P.G. Ciarlet, The Finite Element Method for Elliptic Problems, North-Holland, Amsterdam, 1978.

[5] R.A. DeVore and G.G. Lorentz, Constructive Approximation, Springer-Verlag, Berlin, 1993.

[6] J. Douglas, Jr., F. Furtado, And F. Pereira, On the numerical simulation of waterflooding of heterogeneous petroleum reservoirs, Comput. Geosci., 1 (1997), pp. 155-190.

[7] J. Douglas, Jr., C.-S. Huang, And F. Pereira, The modified method of characteristics with adjusted advection, Numer. Math., 83 (1999), pp. 353-369.

[8] J. Douglas, JR., AND T.F. Russell, Numerical methods for convection-dominated diffusion problems based on combining the method of characteristics with finite element or finite difference procedures, SIAM J. Numer. Anal., 19 (1982), pp. 871-885.

[9] L.C. Evans, Partial Differential Equations, Grad. Stud. Math. 19, American Mathematical Society, Providence, RI, 1998.

[10] R.E. EwIng, ED., The Mathematics of Reservoir Simulation, Frontiers Appl. Math. 1, SIAM, Philadelphia, 1984.

[11] P.A. Farrell, A.F. Hegarty, J.J.H. Miller, E. O’Riordan, and G.I. Shishkin, Robust Computational Techniques for Boundary Layers, Appl. Math. 16, Chapman and Hall/CRC, Boca Raton, FL, 2000. 
[12] R. Helmig, Multiphase Flow and Transport Processes in the Subsurface, Springer-Verlag, Berlin, 1997.

[13] R.J. LeVeque, Finite Volume Methods for Hyperbolic Problems, Cambridge Texts Appl. Math., Cambridge University Press, Cambridge, 2002.

[14] H.-G. Roos, M. Stynes, and L. Tobiska, Numerical Methods for Singularly Perturbed Differential Equations, Springer-Verlag, Berlin, 1996.

[15] H. WANG, An optimal-order error estimate for an ELLAM scheme for two-dimensional linear advection-diffusion equations, SIAM J. Numer. Anal., 37 (2000), pp. 1338-1368.

[16] H. Wang, H.K. Dahle, R.E. Ewing, M.S. Espedal, R.C. Sharpley, and S. Man, An ELLAM Scheme for advection-diffusion equations in two dimensions, SIAM J. Sci. Comput., 20 (1999), pp. 2160-2194.

[17] H. Wang, R.E. Ewing, G. Qin, S.L. Lyons, M. Al-Lawatia, and S. Man, A family of Eulerian-Lagrangian localized adjoint methods for multi-dimensional advection-reaction equations, J. Comput. Phys., 152 (1999), pp. 120-163.

[18] H. Wang, R.E. Ewing, AND T.F. Russell, Eulerian-Lagrangian localized methods for convection-diffusion equations and their convergence analysis, IMA J. Numer. Anal., 15 (1995), pp. 405-459.

[19] H. Wang, D. Liang, R.E. Ewing, S.L. Lyons, and G. Qin, An ELLAM-MFEM solution technique for compressible fluid flows in porous media with point sources and sinks, J. Comput. Phys., 159 (2000), pp. 344-376. 